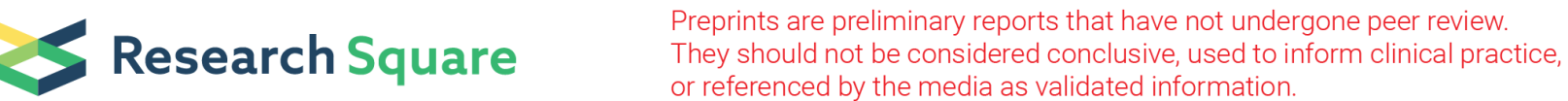

\section{An Injectable Photo-cross-linking Silk Hydrogel System Augments Diabetic Wound Healing in Orthopaedic Surgery Through Spatiotemporal Immunomodulation}

\author{
Jiawei Mei \\ USTC: University of Science and Technology of China \\ Jun Zhou \\ Shanghai Jiao Tong University School of Medicine \\ Lingtong Kong \\ USTC: University of Science and Technology of China

\section{Yong Dai} \\ USTC: University of Science and Technology of China

\section{Xianzuo Zhang} \\ USTC: University of Science and Technology of China

\section{Wenqi Song} \\ Shanghai Jiaotong University: Shanghai Jiao Tong University \\ chen zhu ( $\sim$ zhuchena@ustc.edu.cn) \\ USTC: University of Science and Technology of China
}

\section{Research Article}

Keywords: spatiotemporal immunomodulation, metformin, orthopaedic wound, macrophage polarisation, neutrophil extracellular traps

Posted Date: February 3rd, 2022

DOI: https://doi.org/10.21203/rs.3.rs-1265287/v1

License: (c) (i) This work is licensed under a Creative Commons Attribution 4.0 International License. Read Full License 


\section{Abstract}

Background: The complicated hyperglycaemic and chronic inflammation of diabetic wounds in orthopaedic surgery leads to dysregulated immune cell function and potential infection risk in surgical implants. Immune interventions in diabetic wounds face a possible contradiction between simultaneous establishment of the pro-inflammatory environment in response to potential bacterial invasion and the anti-inflammatory environment required for tissue repair. To study this contradiction and accelerate diabetic-wound healing, we developed a photocurable methacryloxylated silk fibroin hydrogel (Sil-MA) system, co-encapsulated with metformin-loaded mesoporous silica microspheres (MET@MSNs) and silver nanoparticles (Ag NPs).

Results: The hydrogel system (M@M-Ag-Sil-MA) enhanced diabetic-wound healing via spatiotemporal immunomodulation. Sil-MA imparts a hydrogel system with rapid in situ Ultra-Violet-photocurable capability and allows preliminary controlled release of Ag NPs, which can inhibit bacterial aggregation and create a stable, sterile microenvironment. The results confirmed the involvement of Met in the immunomodulatory effects following spatiotemporal dual-controlled release via the mesoporous silica and Sil-MA, which resulted in induction of macrophage polarisation toward the anti-inflammatory M2phenotype. Simultaneously, the M@M-Ag-Sil-MA system inhibited the formation of neutrophil extracellular traps (NETs) and decreased the release of neutrophil elastase, myeloperoxidase, and NETsinduced pro-inflammatory factors. Additionally, the M@M-Ag-Sil-MA system promoted fibroblast migration and endothelial cell angiogenesis in vivo, with verification of enhanced diabetic-wound healing accompanied with the spatiotemporal immunoregulation of macrophages and NETs in a diabetic mouse model.

Conclusions: Our findings demonstrated that the M@M-Ag-Sil-MA hydrogel system resolved the immune contradiction in diabetic wounds through spatiotemporal immunomodulation of macrophages and NETs, suggesting its potential as a promising engineered nano-dressing for the treatment of diabetic wounds in orthopaedic surgery.

\section{Background}

The normal wound-healing process is a complicated series of cellular and biochemical cascade reactions through four ordered sequential but overlapping phases: haemostasis, inflammation, proliferation, and remodelling [1]. However, diabetic wounds fail to heal successfully due to the high-glucose pathological environment, which triggers local immune dysfunction, microangiopathy, and impaired tissue repair, resulting in chronic inflammatory wounds [2,3]. Moreover, diabetic wounds are also at a higher risk of bacterial infection because of skin-barrier disruption, a sustained hyperglycaemic environment, and dysregulated immune cell function [4, 5]. Implant-associated infections due to non-healing diabetic wounds after orthopaedic surgery are devastating and can lead to immediate implant failure, revision surgery, or even amputation due to infection [6-8]. Therefore, manipulation of the complicated inflammatory microenvironment of diabetic wounds may be the most important approach for treating 
this clinical challenge [9]. However, interventions in diabetic wounds are complicated due to the simultaneous necessity to maintain a pro-inflammatory microenvironment capable of controlling bacterial infection and an anti-inflammatory microenvironment required for much needed tissue repair [10]. Specifically, a balance in these microenvironments is complicated by the presence of large amounts of reactive oxygen species (ROS), inflammatory factors, and inflammatory chemokines in diabetic wounds $[11,12]$. ROS clearance and anti-inflammatory immune modulation facilitate wound healing but are detrimental to defence against potential infection $[13,14]$. This suggests that exclusive promotion of anti-inflammatory intervention can indirectly promote potential bacterial invasion, especially in diabetic wounds $[15,16]$. Thus, reconciling the 'immune contradiction' in diabetic wounds may be the most important issue to be addressed.

Metformin (Met) is a classical oral hypoglycaemic agent used for the treatment of type 2 diabetes. Previous studies have shown that Met has good immunomodulatory properties in vivo and in vitro and an especially significant anti-inflammatory capacity in a lipopolysaccharide (LPS)-induced inflammatory environment via Notch1 signalling or AMP-activated protein kinase activation [17]. Exogenous Met can induce macrophages to polarise toward the anti-inflammatory phenotype $M 2$, resulting in the secretion of a series of anti-inflammatory cytokines, such as vascular endothelial growth factor (VEGF) and transforming growth factor- $\beta$, to promote tissue repair $[18,19]$. Mesoporous silicon nanoparticles (MSNs) are porous microspheres with internal mesoporous structures that facilitate the storage and controlled release of drugs [20]. The use of MSN-loaded metformin (MET@MSNs) is an effective drugdelivery method for the controlled release and topical application, with previous studies demonstrating that MET@MSNs exhibit a well-controlled release capacity and immunomodulatory properties for the treatment of tumours [21, 22].

Silk fibroin hydrogel is an injectable hydrogel with good biocompatibility and has been used as a biological scaffold in various cells and tissues [23]. Additionally, silk fibroin hydrogel features a multiporous structure and controlled drug-delivery capability [24]. Following rapid in situ photocuring, methacryloxylated silk fibroin hydrogels (Sil-MA) exhibit extremely high adhesion and sealing properties, making them ideal wound dressings $[25,26]$. Previous studies have demonstrated the effectiveness of silver nanoparticle (Ag NPs)-loaded silk fibroin hydrogels (Ag-Sil-MA) in accelerating wound healing through their antibacterial and promotional collagen-deposition abilities $[27,28]$. Thus, $\mathrm{Ag}$-Sil-MA represents an optimal antibacterial-dressing vehicle with the potential to further load immunomodulatory drugs for the treatment of complicated inflammatory diabetic wounds.

In this study, we employed Ag NP-loaded Sil-MA as an antibacterial-dressing vehicle that was further loaded with MET@MSNs as an immunomodulatory drug (M@M-Ag-Sil-MA). After in situ photocuring in diabetic wounds, bacterial inhibition is initiated by the primary release of Ag NPs from the M@M-Ag-Sil-MA hydrogel system, with macrophage immunomodulation initiated by the dual spatiotemporal slow release of Met to promote M2 polarisation, thereby leading to tissue repair and reconstruction. The results demonstrated a method for spatiotemporal immunomodulation in diabetic 
wounds using different systems to retard drug release and with the prospect of further translation and clinical trials.

\section{Methods}

\section{Preparation and characterization of MET@MSNs}

The synthesis method of MET@MSNs refers to the previous study. Briefly, MSNs (20 mg, XFNANO, China) and Met (60 mg, MACKLIN, China.) were dispersed in ethanol, and then, the mixture was stirred at $37^{\circ} \mathrm{C}$ for $24 \mathrm{~h}$. The mixture was centrifuged at $10,000 \mathrm{rpm}$ for $10 \mathrm{~min}$, the supernatant was discarded, and the pellet was resuspended in ethanol, dried at $80{ }^{\circ} \mathrm{C}$ for $\sim 8 \mathrm{~h}$ for further studies. The drug loading (DL\%) and encapsulation efficiency (EE\%) was determined by UV-Vis spectroscopy (Agilent, Cary 300, USA); the total amount of unloaded MET was calculated at the absorbance of $234 \mathrm{~nm}$. The results were calculated as follows:

Loading efficiency $(\%)=\frac{\text { Mass of total } M E T-\text { Mass of unloaded } M E T(\text { Encapsulated } M E T)}{\text { Mass of total MSNS and encapsulated } M E T} \times$

100 (1)

Encapsulation $\quad$ efficiency

$(\%)$

$=\frac{\text { Mass of total MET }- \text { Mass of unloaded MET(Encapsulated MET })}{\text { Mass of total MET }} \times 100(2)$

To determine the particle size and morphology of the MSN and MET@MSNs, scanning electron microscopy (SEM, Hitachi S 4800, Japan) was performed. The Brunauer-Emmett-Teller (BET) method (ASAP2460, Micromeritics, USA) was employed to assess the structural indicators (surface area, pore volume) related to the mesopores of MSNs and MET@MSNSs. The chemical bond of MSNs and MET@MSNSs were analysed using Fourier transform infrared spectroscopy (FT-IR, Nicolet IS 10, ThermoFisher Scientific, USA).

\section{Preparation and characterization of the hydrogel system}

The Sil-MA (EFL, China) solution was prepared according to the method provided by the manufacturer. Briefly, Sil-MA $(0.5 \mathrm{mg})$ was dissolved in $0.25 \%(\mathrm{w} / \mathrm{v})$ initiator LAP solution $(5 \mathrm{~mL})$ with stirring at room temperature for 30 min. Ag NP solution (100 ppm, XFNANO, China) and MET@MSN with different mass ratios were mixed with Sil-MA solution. The mixture was irradiated under a $405 \mathrm{~nm}$ light source for $\sim 25$ seconds to obtain the hydrogel system including Sil-MA, M@M-Sil-MA, and M@M-Ag-Sil-MA.

The samples were fixed at the sample stage with conductive glue, and the surface was sprayed with gold; finally, the surface morphology was observed using scanning electron microscopy. A rotational rheometer was used to test the rheological properties of the hydrogel system (Discovery HR-2, TA instruments, USA). 
To evaluate the swelling properties of the hydrogel, the samples were weighed and the mass was recorded as $\mathrm{W}_{0}$. PBS was added to the samples at different $\mathrm{pH}$ values $(\mathrm{pH}=6.0 / 7.4 / 8.0)$; the samples were removed at a predetermined time point and weighed after absorbing the residual PBS on the surface of the sample with Kimwipe; the mass was recorded as Wt. The samples were put into PBS again after weighting. The swelling ratio was calculated as follows:

\section{Swelling ratio $(\%)=\frac{w t-w 0}{w 0} \times 100$}

Degradation percent tests were conducted by soaking the samples into PBS with different $\mathrm{pH}$ values at $37^{\circ} \mathrm{C}$ until complete swelling, and then, the initial mass was recorded as Wi. Then, the samples were placed in PBS solution contained collagenase (Biosharp, China) with shaking at $37^{\circ} \mathrm{C}$. At the pre-set timepoints, the samples were taken out and dried with Kimwipe. The remaining mass was recorded as $\mathrm{Wp}$. The degradation percent was calculated by the following formula:

\section{Degradation percent $(\%)=\frac{W i-W p}{W i}$}

M@M-Ag-Sil-MA with different mass ratios of Ag NPs and MET@MSNs (1:1/1:2/1:3) was immersed in 2 $\mathrm{mL}$ PBS with different $\mathrm{pH}$ values $(\mathrm{pH}=6.0 / 7.4 / 8.0)$ to evaluate the cumulative release of components. The release system was incubated at $37^{\circ} \mathrm{C}$ using a shaker $(200 \mathrm{rpm})$. At specific time points, $1 \mathrm{~mL} P B S$ was removed for concentration analysis of Ag NPs and Met, and then, $1 \mathrm{~mL}$ fresh PBS was added into the releasing system. Released Ag NPs and Met were determined by measuring the absorbance at 234 $\mathrm{nm}$ and $400 \mathrm{~nm}$ by UV-Vis spectroscopy, respectively.

\section{Cells isolated and culture}

The femur and tibia medullary cavity of C57/BL6 mice was rinsed with RPMI-1640 medium (Biosharp, China) to obtain primary cells. The neutrophils were isolated according to the steps introduced in the EasySep Mouse Neutrophil Enrichment kit (Stemcell Technologies, Canada). Finally, the neutrophils were cultured in RPMI-1640 medium (Biosharp, China) with 10\% foetal bovine serum (Gibco, USA) and 0.1 $\mathrm{mg} / \mathrm{mL}$ primocin (InvivoGen, USA).

Ea. hy926, L929 and RAW264.7 cells were incubated in high glucose Dulbecco's modified Eagle's medium (DMEM; Gibco, USA), with $10 \%$ foetal bovine serum, $100 \mathrm{U} / \mathrm{mL}$ penicillin, and $100 \mu \mathrm{g} / \mathrm{mL}$ streptomycin.

\section{Verification of Neutrophil Cells}

Neutrophil cells obtained according to the method described above were collected through centrifugation at $300 \times g$ for 10 mins. Cell pellets were then resuspended with $100 \mu \mathrm{L}$ PBS containing $0.25 \mu \mathrm{g}$ APC antimouse/human CD11b antibody (Biolegend, USA) and PE anti-mouse Ly-6G antibody (Biolegend, USA), and then, incubated on ice for 30 min. Finally, the expression levels of CD11b and Ly-6G in neutrophil cells were analysed by flow cytometry using the Beckman CytoFLEX system (Beckman Coulter, USA). 


\section{In vitro cytotoxicity evaluation}

RAW264.7, Ea. Hy926 and L929 cells were used to evaluate cytotoxicity in vitro. The M@M-Ag-Sil-MA and the three cells were co-cultured in six-well plates at $37^{\circ} \mathrm{C}$. Following incubation, M@M-Ag-Sil-MA and the medium were removed and replaced with DMEM containing 10\% CCK-8『Biosharp, China \solution. After incubating for $2 \mathrm{~h}$, the absorbance was measured at $450 \mathrm{~nm}$ using a microplate reader(Bio TEK, Epoch, USA).

Furthermore, RAW264.7 cells were stained with LIVE/DEAD cell Imaging Kit (Invitrogen, USA) about 15 min for the live/dead dyeing test, and Ea. hy926 cells were stained with 100nM TRITC Phalloidin (Yeasen, China) and DAPI (Biosharp, China) to observe cell morphology. Stained cells were observed under an inverted fluorescence microscope (Nikon, ECLIPSE Ts2, Japan).

\section{Conditioned medium and macrophage-conditioned medium Preparation}

Briefly, four samples including Sil-MA, M@M-Sil-MA, Ag-Sil-MA, and M@M-Ag-Sil-MA were soaked into PBS with shaking at $37^{\circ} \mathrm{C}$. According to the results of release of MET and Ag NPs, at the first and seventh day, the PBS solution was collected, centrifuged, filtered using a $0.22 \mu \mathrm{m}$ filter, and mixed with the DMEM at a ratio of 1:2. The mixed medium considered as conditioned medium (CM) were prepared for further testing. For the preparation of macrophage-conditioned medium (MCM), macrophages were cultured with $\mathrm{CM}$ for $24 \mathrm{~h}$. Then, the $\mathrm{CM}$ was replaced with the complete medium DMEM. After another 24-hours incubation, the medium was collected to prepare MCM according to the preparation method of $\mathrm{CM}$.

\section{Formation and extraction of NETs}

Round coverslips were put on the 12-well plates, and isolated neutrophil cells were seeded onto this plate at a concentration of $10^{5}$ cells/well, with the complete medium RPMI- 1640 containing phorbol (Sigma, USA); the samples were incubated for $2 \mathrm{~h}$ at $37^{\circ} \mathrm{C}$. Next, the cells were fixed with $4 \%$ paraformaldehyde for $15 \mathrm{~min}$, and then, washed by PBS three times. The NETs were stained by $0.5 \mu \mathrm{M}$ SYTOX Green Nucleic Acid Stain (Invitrogen, USA) and observed using a confocal laser scanning microscope (ZEISS, LSM710, Germany). For the collection of NET extract, the collecting method was like that for the collection of CM.

\section{In vitro antibacterial test}

S. aureus (ATCC43300) and E. coil (ATCC 35218) cultured in tryptic soy broth (TSB) medium were used to the following antibacterial test.

Bacterial suspensions at a concentration of $10^{7} \mathrm{CFU} / \mathrm{mL}$ were spread on Mueller-Hinton agar plates. SilMA containing different materials (Sil-MA, M@M-Sil-MA, Ag-Sil-MA, M@M-Ag-Sil-MA) were photocured on the curing rings, and then, the rings were placed on the agar plates spread with bacteria and incubated at $37^{\circ} \mathrm{C}$. The diameter of the inhibition zone around the samples was measured. 
The different CMs (Sil-MA, M@M-Sil-MA, Ag-Sil-MA, M@M-Ag-Sil-MA) were co-cultured with bacterial suspension at a concentration of $10^{7} \mathrm{CFU} / \mathrm{mL}$ and incubated at $37^{\circ} \mathrm{C}$. At a predetermined time point, 1 $\mathrm{mL}$ of the suspension was drawn for ten-fold gradient dilution. Subsequently, $100 \mu \mathrm{L}$ of these dilutions was spread onto sheep blood agar plates. After overnight incubation at $37^{\circ} \mathrm{C}$, bacterial colonies on the plates were counted.

To observe the morphology of bacteria, sterile titanium sheets, different $\mathrm{CM}$, and bacterial suspension were co-cultured in six-well plates at $37^{\circ} \mathrm{C}$. The titanium sheets were taken out for SEM testing. In brief, the sheets were fixed with $2.5 \%$ glutaraldehyde overnight at $4{ }^{\circ} \mathrm{C}$. Next, the samples were dehydrated by graded ethanol $(50 \%, 70 \%, 80 \%, 90 \%, 95 \%$ and $100 \%)$ for $10 \mathrm{~min}$ at room temperature. Following freezedrying, the surface of the sheets was sprayed with gold. Finally, the sheets were observed by SEM.

\section{Flow cytometry}

RAW264.7 cells were seeded onto six-well plates with a concentration of $2 \times 10^{5}$ cells/well. After incubation for $24 \mathrm{~h}$ at $37^{\circ} \mathrm{C}$, the medium was removed and washed with PBS three times. Then, different $\mathrm{CM}$ were added into each well. To mimic the in vivo inflammatory conditions of diabetes and explore the effect of NETs and DNase I on the repolarization of macrophages, LPS (Sigma, USA), NETs extract, and DNase I (Sigma, USA) were also added into each well. Following 24-h culturing, RAW264.7 cells were collected via centrifugation at 1,000 rpm for $5 \mathrm{~min}$. Cell pellets were then resuspended with $100 \mu \mathrm{L}$ PBS

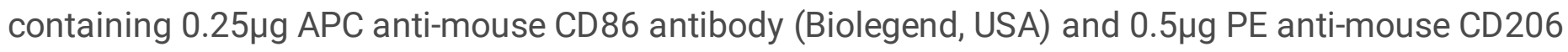
antibody (Biolegend, USA). The samples were then incubated on ice for $30 \mathrm{~min}$. Finally, CD86 and CD206 expression on RAW264.7 cells was analysed by flow cytometry using the Beckman CytoFLEX system.

\section{RT-PCR}

RAW264.7 cells were treated by different CMs for $24 \mathrm{~h}$. iNOS and Arg1 relative expressions were evaluated by RT-PCR. Total RNA was extracted using the EZ-press RNA Purification Kit (EZBioscience, USA). Purified RNA was reverse transcribed into cDNA using a Colour Reverse Transcription Kit (EZBioscience, USA). Next, RT-qPCR was performed using a $2 \times$ Colour SYBR Green qPCR Master Mix (EZBioscience, USA). Gene expressions were calculated using the $2^{-\Delta \triangle C t}$ method. Primer sequences of Gapdh, iNOS and Arg-1 are shown in Table S1.

\section{ELISA}

After 24-h incubation with the same method as above mentioned, the cultured medium was collected and centrifuged at 3,000 rpm for $20 \mathrm{~min}$. The supernatant was used to determine the inflammatory cytokine expression by Elisa Kits (Dakewe Biotech, China) according to the manufacturer's protocol.

\section{Tube formation}

Briefly, Ea. hy 926 cells $\left(10^{4}\right.$ cells/well) were pre-treated with different MCM for $24 \mathrm{~h}$ were seeded onto $\mu$ slide plates (IBIDI, Germany) pre-coated with Matrigel matrix (BD, Corning, USA). After incubation for $6 \mathrm{~h}$ 
at $37^{\circ} \mathrm{C}$, the tube formation was fixed and stained by 100nM FITC phalloidin (Yeasen, China). The tubes were then observed using an inverted fluorescence microscope. The numbers of junctions and circles were counted manually.

\section{Scratch Assay}

L929 cells $\left(2 \times 10^{5}\right.$ cells/dish) were seeded in $35 \mathrm{~mm}$ cell culture dishes and incubated at $37{ }^{\circ} \mathrm{C}$ to $90 \%$ confluence. Subsequently, $200 \mu \mathrm{L}$ pipet tips were used to draw a line at the bottom of the dishes and washed three times with PBS. Next, the cells were co-cultured with MCM of five groups for $24 \mathrm{~h}$ at $37^{\circ} \mathrm{C}$. At 0 and $24 \mathrm{~h}$, the cells in each group were fixed, washed, stained with crystal violet for $3 \mathrm{~min}$, and observed using an optical microscope. Finally, the migration rates in various groups were assessed using Image J software (NIH, Version 1.52, USA).

\section{Inhibition of Nets}

To explore the inhibition of Met on NETs formation in the infection microenvironment of diabetes, neutrophils were cultured with the CM of three groups containing glucose or LPS for two hours. Finally, the cells were stained with $1 \mu \mathrm{M}$ SYTOX Green Nucleic Acid Stain and observed using a confocal laser scanning microscope.

\section{STZ-Induced Diabetic Mice}

In this research, all animal experiments were approved by Animal Welfare Ethics Committee of The First Hospital Affiliated University of Science and Technology of China.

C57BL/ 6 mice (6-8 weeks, 23-26 g) were used to induce diabetes. In brief, $100 \mathrm{mg} / \mathrm{kg}$ streptozotocin (STZ) (Sigma, USA) was injected intraperitoneally into the mice fasted with food and water for one day before injection. The blood glucose levels were determined using glucose meters (Roche, Germany), and all mice with glycaemia $\geq 16.7 \mathrm{mmol} / \mathrm{L}$ were considered diabetic.

\section{In-vivo wound healing evaluation}

We randomly divided the 45 diabetic mice into five groups: control, Sil-MA, M@M-Sil-MA, Ag-Sil-MA, and M@M-Ag-Sil-MA. These diabetic mice were anesthetized by inhalation gas anaesthesia. After the dorsal skin of mice was shaven and disinfected, a pouch with a diameter of $8 \mathrm{~mm}$ was used to create a skin wound; 0.2 mL hydrogel (Sil-MA, M@M-Sil-MA, Ag-Sil-MA, and M@M-Ag-Sil-MA) was dropped onto the wound, and then, the materials were photocured under $405 \mathrm{~nm}$ ultraviolet light for $25 \mathrm{~s}$. At days 0, 3, 7, 10, and 14 after surgery, the condition of the wounds was recorded using a digital camera to assess the wound healing rate, and the weight were also recorded.

\section{Histological Analysis}


At days $1,3,7$, and 14 after surgery, some mice were euthanized in each group. The wound skin tissues were collected and fixed in $4 \%$ paraformaldehyde, dehydrated using graded ethanol, embedded in paraffin wax, and cut into sections by RM2016 Microtome (Leica, Germany). Some sections were stained by H\&E (Solarbio, China) and Masson (Solarbio, China) staining to assess the degree of inflammatory cell infiltration, collagen deposition, and bacterial infection. For immunofluorescence staining, primary CD86 (1:3,000, Bioss, China), CD206 (1:400, Servicebio, China), myeloperoxidase (MPO, 1:400, Servicebio, China), citrullinated histone3 ( $\mathrm{CitH} 3,1: 3,000, \mathrm{Abcam}$, UK), secondary HRP conjugated Goat Anti-Rabbit $\lg G(H+L)$ (1:500, Servicebio, China), and Alexa Fluor ${ }^{\circledR}$ 488-conjugated Goat Anti-Rabbit $\lg G(H+L)$ (1:400, Servicebio, China) antibodies were used to observe the inflammatory response degree, macrophage phenotype, and NETs in wound skin tissues. For immunohistochemistry, to observe angiogenesis in wound skin tissues, the sections were incubated with the following primary antibodies: mouse anti-alpha smooth muscle Actin antibody (1:300, Bioss, China) and rabbit anti-VEGF antibody (1:200, Bioss, China) at $4{ }^{\circ} \mathrm{C}$ overnight, and further incubated with the following secondary antibodies: HRP-conjugated goat anti-mouse lgG $(H+L)$ (1:200, Servicebio, China) and HRP-conjugated goat anti-rabbit lgG $(H+L)(1: 200$, Servicebio, China) for $1 \mathrm{~h}$. The binding sites were then visualized with a DAB detection kit (DAKO, Denmark), counterstained with haematoxylin (Servicebio, China), and mounted with neutral resinto (Servicebio, China). All stained sections were observed using a microscope (Nikon Ci-S, Japan).

\section{Biocompatibility Evaluation in vivo}

The major organs (heart, liver, spleen, lung, and kidney) from each group were collected, fixed, dehydrated, embedded, cut, and stained with H\&E dye. The sections were observed by optical microscope to assess the biocompatibility of samples in vivo.

\section{Statistical Analysis}

All data were exhibited as the mean \pm standard deviation and analysed using GraphPad Prism software (version 8.0) and Origin (Version 2019b). Statistically significant values were assessed using two-sided student's $t$ test and one-way analysis of variance (ANOVA) test. $P$ value $<0.05$ was considered statistically significant.

\section{Results And Discussion}

\section{Characterisation of MET@MSNSs and M@M-Ag-Sil-MA}

After synthesizing porous MET@MSNs nanospheres according to a previously described method [22], we evaluated the morphological characteristics of MSN and MET@MSNs using scanning electron microscopy (SEM) (Fig. 1a, b). Our results indicated that the silica NPs showed a regular spherical morphology with good dispersion and homogeneity and an average particle size of $\sim 85.81 \mathrm{~nm}$. Notably, we detected significant mesopores on the surface of the drug-free particles, with MET@MSNs exhibiting a larger size (average diameter: $\sim 90.71 \mathrm{~nm}$ ) and no significant mesopores on the surface. This result was similar with that of a previous study [21,22]. Furthermore, we determined the specific surface areas of the 
MSNs and MET@MSNs using an N2 adsorption analyser, with the pore-size distribution and surface area of the MET@MSNs determined by Barrett-Joyner-Halenda and Brunauer-Emmett-Teller (BET) procedures, respectively (Fig. 1C, d). The results showed that the BET specific surface area of the unloaded monodisperse silica microspheres was $307.20 \mathrm{~m}^{2} / \mathrm{g}$ with a pore volume of $8.73 \mathrm{~nm}$, whereas the specific surface area and pore size of the MET@MSNs were reduced $\left(281.11 \mathrm{~m}^{2} / \mathrm{g}\right.$ and $8.48 \mathrm{~nm}$, respectively). Fourier-transform infrared spectroscopic results of monodisperse silica microspheres (Fig. 1e) revealed the main absorption bands at 2,695, 2,217, and $1,475 \mathrm{~cm}^{-1}$ belonging to the $\mathrm{C}-\mathrm{H}-$ stretching vibration and $\mathrm{C}-\mathrm{H}$ deformation, respectively. Moreover, we attributed the absorption peaks at 1,168 and $1,064 \mathrm{~cm}^{-1}$ to the external and internal asymmetric stretching vibrations of the $\mathrm{Si}-\mathrm{O}-\mathrm{Si}$ bond. We detected typical bands at 1,485 and $1,084 \mathrm{~cm}^{-1}$ after Met loading, which were attributed to $-\mathrm{C}=\mathrm{N}$ and $\mathrm{C}=\mathrm{N}$ Schiff base stretching. The $\mathrm{N}-\mathrm{H}$-stretching vibrations at $3,321 \mathrm{~cm}^{-1}$ and $\mathrm{N}-\mathrm{H}$-deformation vibrations at $2,854 \mathrm{~cm}^{-1}$ indicated the absorption of amino groups by dispersed monodisperse NPs.

A previous study reported the optimal concentration of Ag NPs colloids loaded in hydrogels for antimicrobial and wound-healing promotion [29]. In the present study, we used the same concentration ratios of Ag NPs colloid concentrations as antimicrobial agents for the hydrogel system. Different ratios of the MET@MSNs aqueous dispersion and stationary terminal concentrations of Ag NPs colloids were incorporated into the Sil-MA hydrogel-precursor solution, and photocuring was performed by UV radiation at $405 \mathrm{~nm}$ in the presence of a photo initiator (lithium phenyl-2,4,6-trimethylbenzoylphosphinate, LAP). Synthesis of M@M-Ag-Sil-MA (Fig. 2a) revealed a photocured hydrogel that maintained its morphology in an inverted or horizontal position and demonstrated good adhesion, deformation, and writing abilities at the finger joints (with or without nitrile gloves) and on hairy skin with or without nitrile gloves when inverted (Fig. 2b and S1-S2). SEM assessment (Fig. 2c) of the surface morphologies of the developed hydrogel system resulted in micrographs showing the interconnected porous meshwork of Sil-MA and M@M-Ag-Sil-MA scaffolds with void sizes $>100 \mu \mathrm{m}$, which encouraged cell adhesion, proliferation, and migration within the hydrogels. Additionally, rheological data confirmed the successful preparation of the M@M-Ag-Sil-MA hydrogel system, with the storage modulus $(G)$ surpassing the loss modulus (G") immediately after activation by $405 \mathrm{~nm}$ UV radiation and lasting for $100 \mathrm{~s}$ and promoting the rapid in situ photo curability of the hydrogel system (Fig. 2d). However, upon the stable formation of hydrogels, $\mathrm{G}$ was independent of the shear frequency from $50 \mathrm{~Hz}$ to $250 \mathrm{~Hz}$, indicating that the hydrogel system was robust and suitable for application as a wound dressing (Fig. 2e). To demonstrate the ability of this system to absorb tissue fluid, testing of the swelling effect of the hydrogel system at different $\mathrm{pH}$ values revealed a swelling rate of $200 \%$ (Fig. $2 \mathrm{f}-\mathrm{h}$ ) and an ability to maintain a limited swelling ratio at different $\mathrm{pH}$ values ( $\mathrm{pH}=6.0 / 7.4 / 8.0)$, with no significant changes observed due to drug loading. Our findings suggested the potential stability of the M@M-Ag-Sil-MA hydrogel system in infected wound applications according to its compatibility with the wound area without over-swelling. Further observations indicated that the degradation of the hydrogel system continued until day 16 , with the degradation time slightly faster in acidic $(\mathrm{pH}=6.0)$ and alkaline $(\mathrm{pH}=8.0)$ environments than in neutral $(\mathrm{pH}=7.4)$ environments (Fig. $2 \mathrm{i}-\mathrm{k})$. The degradation time of all hydrogels exceeded two weeks, which is sufficient for the time required for 
wound healing. These results indicate that the hydrogel characteristics suggest their suitability as wound dressings for orthopaedic patients with diabetes.

\section{Dual-controlled drug release and biocompatibility of the M@M-Ag-Sil-MA hydrogel in vitro}

We then determined the release of Ag NPs and Met from the M@M-Ag-Sil-MA hydrogel system by UVvis spectroscopy. Specifically, hydrogel systems with different Ag NPs and MET@MSNs mass ratios (1:1, 2:1 and 3:1) were immersed in liquid environments with different $\mathrm{pH}$ values $(\mathrm{pH}=6.0 / 7.4 / 8.0)$ to observe the cumulative concentration of the components in the environment. At a mass ratio of $1: 1$, the cumulative release concentration of Ag NPs was consistently higher than the cumulative release concentration of Met, while at a mass ratio of 3:1, the cumulative release concentration of Met was consistently higher than the cumulative release concentration of Ag NPs. When the mass ratio was 2:1, the cumulative release concentration of Ag NPs was higher than the cumulative release concentration of Met by day 5 , whereas the cumulative release concentration of Met was consistently higher than the cumulative release concentration of Ag NPs after day 7 (Fig. 3a-c). The release of the hydrogel system was accelerated in acidic (Fig. 3d-f) and alkaline environments (Fig. S3), whereas the release order of Ag NPs and Met have no alteration.

Furthermore, we determined the biocompatibility of the M@M-Ag-Sil-MA hydrogel system with cells. First, fluorescence microscopy observations after co-culture of Ea. hy 926 cells with M@M-Sil-MA ${ }_{1}$ and M@M-Sil-MA 7 harbouring different Ag NPs and MET@MSNs mass ratios showed favourable cytoskeletal morphology (Fig. 3g). Live/dead assays showed that RAW264.7 cells were viable on M@M-Ag-Sil-MA, with $>90 \%$ viability after co-culture with all $\mathrm{M} @ \mathrm{M}-\mathrm{Sil}_{-\mathrm{MA}}$ and $\mathrm{M} @ \mathrm{M}-\mathrm{Sil}-\mathrm{MA}_{7}$ (the $\mathrm{CM}$ of hydrogel for day 1 and 7, respectively) (Fig. 3h). Quantification of L929 cell viability by the Cell Counting Kit-8 assay after co-culture with all M@M-Sil-MA ${ }_{1}$ and $\mathrm{M} @ \mathrm{M}-\mathrm{Sil}-\mathrm{MA}_{7}$ (Fig. 3i, j) indicated similar optical density values in all groups with no statistical differences. These results of the drug-release experiments and cytocompatibility assays demonstrated the controlled drug release and safety of the M@M-Ag-Sil-MA hydrogel system. To illustrate the spatiotemporal modulation of the hydrogel system, we chose hydrogels with a mass ratio of MET@MSNs to Ag NPs of 2:1 for subsequent experiments.

\section{Antibacterial performance of the M@M-Ag-Sil-MA hydrogel in vitro}

We then evaluated the ability of preliminary release of the Ag NPs incorporated into the M@M-Ag-SilMA hydrogel system to inhibit bacterial infection. The relative colony counts were $7.72 \pm 0.10(\mathrm{CFU} / \mathrm{mL})$ for Staphylococcus aureus (S. aureus) and $7.15 \pm 0.09$ (CFU/mL) for Escherichia coli (E. Coli) when cocultured with $\mathrm{CM}$ of Sil-MA, which were decreased significantly when co-cultured with the $\mathrm{CM}$ of $\mathrm{Ag}-\mathrm{Sil}-$ MA and M@M-Ag-Sil-MA hydrogel system, leaving only $6.90 \pm 0.09(\mathrm{CFU} / \mathrm{mL})$ and $6.30 \pm 0.43$ $(\mathrm{CFU} / \mathrm{mL}$ ) of $S$. aureus and E. coli, respectively, in the M@M-Ag-Sil-MA hydrogel system group (Fig. 4a$\mathrm{c}$ and S4). Additionally, zone of inhibition (ZOI) tests of the hydrogel system against $S$. aureus and $E$. Coli revealed similar ZOls for the Ag-Sil-MA and M@M-Ag-Sil-MA hydrogels to those of antibiotic-sensitive tablets but better than the Sil-MA and M@M-Sil-MA hydrogels and the blank control tablet (Fig. 4d-f and 
S5). Despite the limited contact area of the hydrogel with bacteria, the superior porous structure and water content of the hydrogel system conferred the ability to diffuse Ag NPs. The diffusion of $\mathrm{Ag}^{+}$from the Ag NPs provided the hydrogel system with sustained bacterial inhibition, a process that was accompanied by the degradation of the hydrogel system [30]. Furthermore, SEM images (Fig. $4 \mathrm{~g}$ and S6) showed that bacteria could form an intact and dense biofilm when co-cultured with the CM of Sil-MA and M@M-Sil-MA hydrogels, whereas we observed only sporadic non-biofilm-forming and ruptured bacteria after co-culture with the $\mathrm{CM}$ of Ag-Sil-MA hydrogel and M@M-Ag-Sil-MA hydrogel system. These results confirmed that the antimicrobial activity of the M@M-Ag-Sil-MA hydrogel by releasing Ag NPs, which provided a continuous environment that promoted bacterial inhibition for wound healing and permitted the subsequent transition from a pro-inflammatory to an anti-inflammatory phenotype [31].

\section{Evaluation of macrophage polarisation and function in vitro}

We then evaluated the effect of the controlled release of MET following bacterial inhibition by release of Ag NPs. To mimic the in vivo inflammatory conditions of diabetes, we treated RAW264.7 cells with LPS to induce macrophage polarisation to a pro-inflammatory M1 phenotype [32, 33]. At 6-hours post-induction, the medium was replaced with a new medium containing either LPS (LPS group) or CM (for days 1 and 7) of Sil-MA, M@M-Sil-MA, Ag-Sil-MA, or M@M-Ag-Sil-MA hydrogels, followed by culture for 1 day and evaluation of macrophage polarisation. Flow cytometry and RT-PCR assay (Fig. 5a-c) results showed that all cultures with the respective CM (from 1-day cultures) showed similar results to the LPS group in terms of elevated expression of the M1 phenotype marker CD86 and inducible nitric oxide synthase (iNOS) [34, 35]. By contrast, cultures with CM from 7-days cultures (M@M-Sil-MA 7 and M@M-Ag-Sil$M_{7}$ ) reduced CD86 and iNOS expression but increased the expression of the M2 phenotype marker CD206 and arginases-1 (Arg-1) relative to levels observed in the LPS, Sil-MA ${ }_{7}$, and Ag-Sil-MA 7 groups [36]. To detect the representative cytokines secreted by M1 and M2 macrophages after spatiotemporal immunoregulation by the $\mathrm{M} @ \mathrm{M}-\mathrm{Ag}-\mathrm{Sil}-\mathrm{MA} \mathrm{A}_{7}$ hydrogel system, we measured the concentrations of tumour necrosis factor (TNF)- $a$ and IL-10 by enzyme-linked immunosorbent assay (ELISA) (Fig. 5d, e). Cells treated with LPS and CM of M@M-Sil-MA,$A g-S i l-M_{1}$, and M@M-Ag-Sil-MA, secreted similar levels of TNF- $a$, which is a representative pro-inflammatory factor produced by $\mathrm{M} 1$ macrophages. By contrast, RAW 264.7 cells treated CM of M@M-Sil-MA 7 and M@M-Ag-Sil-MA ${ }_{7}$ secreted higher levels of the anti-inflammatory cytokines IL-10, which are mainly produced by M2 macrophages. During diabeticwound healing, the polarization of M2 macrophages contribute to limited pro-inflammatory cytokine release in the inflammatory microenvironment, as well as the release of repair cytokines for angiogenesis and cell migration, which are required for tissue repair. M@M-Ag-Sil-MA hydrogel accomplished immunomodulation of M2 polarization through a dual-controlled release of the immunomodulator Met, without affecting the level of inflammation in the pre-wound healing antimicrobial environment.

Assessment of the proangiogenic potential of the M@M-Ag-Sil-MA hydrogel via tube-formation assays (Fig. 5f) revealed that none of the endothelial cells co-incubated with day-1 MCM for 6 hours on the Matrigel substratum showed significant tube formation trends, whereas we observed the densest tubular 
networks in the groups co-incubated with $\mathrm{M} @ \mathrm{M}-$ Sil-MA $\mathrm{MCO}_{70}$ and $\mathrm{M} @ \mathrm{M}-\mathrm{Ag}-\mathrm{Sil}-\mathrm{MA}_{7 C_{0}}$. Moreover, these groups exhibited more circles $(18.00 \pm 4.58 \%$ and $18.00 \pm 7.00 \%)$ and junctions $(49.33 \pm 9.30 \%$ and 49.00 $\pm 9.00 \%$ ) relative to those observed in the other groups (Fig. $5 \mathrm{~h}, \mathrm{i}$ ).

We then assessed the in vitro ability of the $\mathrm{M} @ \mathrm{M}-\mathrm{Ag}$-Sil-MA hydrogel to promote tissue regeneration via macrophage polarization modulation according to fibroblast migration and tube formation by endothelial cells. Scratch assays (Fig. 5g) using mouse L929 cells co-incubated with different MCMs (days 1 and 7) indicated that $L 929$ cells migrated significantly faster when co-incubated with MCM of M@M-Sil-MA $7 C_{0}$ and $\mathrm{M} @ \mathrm{M}-\mathrm{Ag}-\mathrm{Sil}_{\mathrm{M}} \mathrm{MA}_{7 \mathrm{CO}_{0}}$ relative to those incubated with other day-7 and all day-1 MCM, with quantified data (Fig. $5 \mathrm{j}$ ), clearly demonstrating a migration ratio of $39.88 \pm 2.42 \%$ for $\mathrm{M} @ \mathrm{M}-\mathrm{Sil}-\mathrm{MA}_{7 \mathrm{Co}_{0}}$ and 41.46 $\pm 1.50 \%$ for $\mathrm{M} @ \mathrm{M}-\mathrm{Ag}-\mathrm{Sil}-\mathrm{MA}_{7 \mathrm{C}_{o}}$ as compared with $6.48 \pm 1.08 \%$ for Sil-MA $\mathrm{M}_{7 C_{o}}$ and $9.57 \pm 1.68 \%$ for $\mathrm{Ag}-$ Sil-MA $7 C_{0}(p<0.001)$. These results indicated that the M@M-Ag-Sil-MA hydrogel may have promoted fibroblast migration through the release of Met and the inherent repair ability of silk fibroin.

These findings revealed that the $\mathrm{M} @ \mathrm{M}-\mathrm{Ag}$-Sil-MA hydrogel system was unable to modulate the antiinflammatory polarisation of macrophages after a 1-day co-culture, whereas on day 7, we observed altered M2 polarisation. This process is correlated with the dual-controlled release of Met. Moreover, the spatiotemporal modulation of macrophage polarisation mimics the immune response associated with normal wound healing, which avoids the potential risk of infection caused by immune intervention and allows macrophages to promote tissue repair and angiogenesis in a stable, sterile environment.

\section{Evaluation of inhibited neutrophil extracellular traps (NETs)formation in vitro}

The formation of large numbers of NETs in diabetic wounds is a cause of delayed wound healing and leads to persistent inflammation [37]. Both a hyperglycaemic microenvironment and bacterial invasion are potential mechanisms for NETs formation in diabetic wounds [38]. Normal NETs formation is programmed to be pro-inflammatory and bactericidal; however, in the uncontrolled inflammatory microenvironment of diabetic wounds, this process is derailed by the continuous recruitment and infiltration of neutrophils and NETosis [39]. Therefore, inhibition of NETs formation might represent a potential way to promote wound healing, and previous studies report that Met demonstrates significant inhibition of NETs formation [40, 41].

To evaluate the ability of the hydrogel system to inhibit NETs formation, we first established an in vitro model of the NETs formation conditions found in diabetic wounds. Isolated mouse bone marrow neutrophils were activated in vitro by treatment with LPS and high glucose medium ( $50 \mathrm{mM})$ to induce NETs formation, followed by co-incubation with CM of 1-day cultures of each respective hydrogel system. SYTOX green staining to visualise extracellular DNA (Fig. 6a) revealed fluorescence micrographs that showed similar high NETs generation, whereas co-incubation of induced neutrophils (High glucose/LPS) with $\mathrm{CM}$ of $\mathrm{M} @ \mathrm{M}-$ Sil-MA 7 and $\mathrm{M} @ \mathrm{M}-\mathrm{Ag}-$ Sil-MA 7 cultures showed a clear reduction in NETs formation. We subsequently confirmed this result by fluorescence intensity quantification (Fig. 6b, S7). Furthermore, we observed elevated levels of NETs components, such as neutrophil elastase (NE) and myeloperoxidase 
(MPO) [42], in all groups incubated with 1-day extracts, whereas these levels were significantly lowered following incubation with M@M-Sil-MA 7 and $\mathrm{M} @ M-A g-S i l-M A_{7}$ extracts (Fig. 6c, d and S8).

To further investigate the effect of hydrogels on macrophage activity via inhibited NETs formation, we cocultured RAW264.7 cells with medium extracts of NETs formed by high glucose or medium extracts of NETs inhibited by co-incubation with CM of M@M-Ag-Sil-MA , with LPS-containing medium and extracts of DNase I-treated NETs serving as controls. Flow cytometry (Fig. 6e) results showed a significant repolarisation to the M1 phenotype after treatment with LPS and high-glucose NETs extract, whereas the M2 phenotype was sustained in the presence of the M@M-Ag-Sil-MA,-stimulated NETs extract. Surprisingly, we also observed M1 repolarisation in the presence of the DNase I-treated NET extract, with a possible explanation being that neutrophils also release pro-inflammatory factors during NETosis. Furthermore, ELISA experiments to assess cytokine levels confirmed significant reductions in pro-inflammatory factors, such as TNF-a, and increases in IL-10, a pro-inflammatory factor, following coincubation with $\mathrm{M} @ \mathrm{M}-\mathrm{Ag}-\mathrm{Sil}-\mathrm{MA} \mathrm{A}_{7}$. These results (Fig. 6f-i) suggested that the M@M-Ag-Sil-MA hydrogel demonstrated a significant immunomodulatory effect on NETs formation via the dualcontrolled-release of Met. This regulatory process revealed direct NETs-suppressive and antiinflammatory activities that sustained the anti-inflammatory polarization of macrophages $[43,44]$.

\section{M@M-Ag-Sil-MA hydrogel promotes wound healing in vivo}

Following the establishment of a mouse model of type-2 diabetes (blood glucose level: $16.7 \mathrm{mM}$ ) and harbouring a full-sized back wound (diameter: $8 \mathrm{~mm}$ ) according to previous studies, we injected the respective hydrogel systems (Sil-MA, M@M-Sil-MA, Ag-Sil-MA, and M@M-Ag-Sil-MA) in situ into the wound, followed by UV irradiation at $405 \mathrm{~nm}$ to promote photocuring and attachment to the wound surface (Fig. 7a). All hydrogel-attached wounds were left uncovered, with saline administration to the wound site used as the control. All wounds were photographed using a digital camera to measure the healing that occurred at days $0,3,7,10$, and 14 after surgery, and the gradual shrinkage of wound dimensions was quantified using ImageJ software. The results showed that all wounds to which each of the hydrogels were administered displayed noticeable shrinkage of the wound area during the regeneration stage and relative to the control group (Fig. 7b, c). Specifically, the group administered M@M-Ag-Sil-MA showed the fastest healing rate as compared with all other hydrogel groups. During the early stages of wound healing, M@M-Sil-MA-treated wounds showed marked redness and swelling, which we attributed to direct anti-inflammatory immunomodulation. Similarly, Ag-Sil-MA- and M@MAg-Sil-MA-treated wounds exhibited increased drying and shrinkage, possibly due to the inhibition of bacterial infection following the release of Ag NPs and the release of Met, which also reduced scar formation.

Moreover, haematoxylin and eosin (H\&E) staining of skin surrounding the wound at days 1, 3, 7, and 14 after surgery showed a markedly higher healing rate in the M@M-Ag-Sil-MA-treated group relative to the other groups (Fig. 7f). Specifically, all groups showed significant inflammatory leukocyte infiltration according to H\&E staining on day 1, and the Ag-Sil-MA and M@M-Ag-Sil-MA groups showed 
significantly better inflammatory cell infiltration on day 3 . Notably, H\&E staining results on days 7 and 14 clearly showed complete squamous epithelium, well-distributed neovascularisation, new collagen fibre formation, and sebaceous glands in the M@M-Ag-Sil-MA group, indicating the excellent tissue-repair capacity of this hydrogel. To evaluate the collagen formation in different hydrogel-treated wounds, we also performed Masson's trichrome staining at the same post-surgery time points (Fig. 7g). On days 1 and 3 , all wounds harboured granulation tissues with regular re-epithelialisation and few signs of inflammation in M@M-Ag-Sil-MA-treated wounds, whereas on day 14, we observed extensive collagen deposition and dense, wavy collagen fibres in the same wounds along with a more mature newly formed structure that closely resembled normal tissue. Our findings confirmed the inhibition of bacterial invasion, as well as the accelerated granulation tissue formation, dense collagen deposition, and enhanced wound healing efficacy of the M@M-Ag-Sil-MA hydrogel.

\section{M@M-Ag-Sil-MA hydrogel promotes spatiotemporal immunomodulation in vivo}

We then performed immunofluorescence staining of wound tissues to evaluate the spatiotemporal immunomodulation of diabetic wounds in vivo using the M@M-Ag-Sil-MA hydrogel. We consistently observed staining of CD86 and CD206 in wounded tissues and representative markers of the proinflammatory M1 and anti-inflammatory M2 phenotypes of macrophages (Fig. 8a). On days 1 and 3, we observed a high number of M1 macrophages (CD86+CD206-) in all hydrogel-treated groups, as well as in the control group. Notably, the number of M2 macrophages (CD86-CD206+) in the M@M-Ag-Sil-MAtreated group increased on day 7 , whereas tissues from the control and other hydrogel-treated groups showed a persistent pro-inflammatory M1 phenotype, indicating unaltered macrophage polarisation. We continued to observe the same trends on days 14, with the M@M-Ag-Sil-MA-treated group showing a higher density of M2 macrophages than the other groups. However, on day 14, both M1 and M2 macrophage counts in M@M-Ag-Sil-MA-treated wounds decreased relative to those on day 7, which indicated normalised tissue regeneration and decreased involvement of macrophage-mediated tissue repair.

Furthermore, we confirmed the inhibition of NETs formation in M@M-Ag-Sil-MA-treated diabetic wounds by immunofluorescence staining of the NETs components citrullinated histone 3 (CitH3) and MPO (Fig. 8b). As expected, the M@M-Ag-Sil-MA-treated group showed high NET formation ( $\mathrm{CitH} 3+\mathrm{MPO}+$ ) in the first $3 \mathrm{~d}$ following surgery and significant NET inhibition from day 7 onwards, whereas the control and other hydrogel-treated groups maintained high NET formation and infiltration. Notably, the group treated with M@M-Sil-MA, which also contained Met, did not demonstrate an ability to promote macrophage polarisation to the anti-inflammatory phenotype or inhibit NETs formation in vivo. This might be attributed to a more complicated inflammatory microenvironment of diabetic wounds in vivo, where aggressive anti-inflammatory intervention is incapable of rescuing intrinsic immune functions. These results confirmed that the spatiotemporal immunomodulation by M@M-Ag-Sil-MA was due to its ability to mimic the physiological processes associated with normal wound healing by providing a stable immune microenvironment for angiogenesis and collagen deposition required for tissue regeneration. 
We then determined M@M-Ag-Sil-MA-mediated changes in the rate of angiogenesis in diabetic wounds according to immunohistochemical (IHC) analysis of two markers of vascular formation (VEGF) and asmooth muscle actin (a-SMA) $[45,46]$. IHC staining revealed gradual increases in the number and density of blood vessels in the M@M-Ag-Sil-MA-treated group from days 3 to 14, with both of these measurements higher than those in the control and other hydrogel groups (Fig. 8c, d). Interestingly, we observed high levels of both VEGF and a-SMA during the first $7 \mathrm{~d}$ post-surgery in the M@M-Ag-Sil-MAtreated wound, whereas on day 14, there was a slight decrease in VEGF expression. This might be explained by the stabilisation of neovascular alterations during the wound-regeneration process toward the end stages of healing. Furthermore, assessment of in vivo biocompatibility in mice 14 days after surgery by H\&E staining of the heart, liver, lungs, spleen, and kidneys confirmed the non-toxicity of the M@M-Ag-Sil-MA hydrogel (Fig. S9).

\section{Conclusion}

In this study, we synthesized and characterized an injectable M@M-Ag-Sil-MA hydrogel system that can be photocured in situ on diabetic wounds. This M@M-Ag-Sil-MA hydrogel system promotes tissue repair and angiogenesis by first-phase bacterial inhibition followed by the dual-controlled release of Met for spatiotemporal immunomodulation of macrophages and NETs formation. These findings demonstrated that the M@M-Ag-Sil-MA hydrogel system resolved the immune contradiction in diabetic wounds through a two-step spatiotemporal immunomodulation, suggesting its potential as a promising engineered nano-dressing for the treatment of diabetic wounds in orthopaedic surgery.

\section{Abbreviations}

a-SMA, a-smooth muscle actin

Ag, silver

BET, Brunauer

Emmett-Teller

CitH3, citrullinated histone 3

$\mathrm{CM}$, conditioned medium

MCM, macrophage-conditioned medium

ELISA, enzyme-linked immunosorbent assay

$\mathrm{IHC}$, immunohistochemical

IL, interleukin

LPS, lipopolysaccharide

Met, Metformin

MPO, myeloperoxidase

MSN, mesoporous silica microsphere

NET, neutrophil extracellular trap 
NP, nanoparticle

ROS, reactive oxygen species

SEM, scanning electron microscopy

Sil-MA, Methacryloxylated silk fibroin hydrogel

TNF, tumour necrosis factor

ZOI, zone of inhibition

\section{Declarations}

\section{Ethics approval and consent to participate}

The animal experiments were approved by the Animal Care Committee of the First Affiliated Hospital of University of Science and Technology of China.

\section{Consent for publication}

Not applicable.

\section{Availability of data and materials}

The datasets used and/or analysed during the current study are available from the corresponding author on reasonable request.

\section{Competing interests}

The authors declare that they have no competing interests.

\section{Funding}

This work was supported by the National Natural Science Foundation of China (Grant No. 81871788) and the Anhui Provincial Postdoctoral Science Foundation (Grant No. 2019B302).

\section{Authors' contributions}

JM, JZ and LK carried out experiments and wrote the paper. XZ, WS and CZ designed the study. JM and JZ contributed to sample preparation. JZ, YD and JM provided guidance and assistance on relevant biological information and experiments. All authors approved the final manuscript.

\section{Acknowledgements}

None.

\section{References}


1. Boateng J, Catanzano O. Advanced Therapeutic Dressings for Effective Wound Healing--A Review. J Pharm Sci. 2015;104(11):3653-80.

2. Frykberg RG, Banks J. Challenges in the Treatment of Chronic Wounds. Adv Wound Care (New Rochelle). 2015;4(9):560-82.

3. Davis FM, Kimball A, Boniakowski A, Gallagher K. Dysfunctional Wound Healing in Diabetic Foot Ulcers: New Crossroads. Curr Diab Rep. 2018;18(1):2.

4. Geng K, Ma X, Jiang Z, Huang W, Gao C, Pu Y, Luo L, Xu Y, Xu Y. Innate Immunity in Diabetic Wound Healing: Focus on the Mastermind Hidden in Chronic Inflammatory. Front Pharmacol. 2021;12:653940.

5. Brazil JC, Quiros M, Nusrat A, Parkos CA. Innate immune cell-epithelial crosstalk during wound repair. J Clin Invest. 2019;129(8):2983-93.

6. Chen W, Klemt C, Smith EJ, Tirumala V, Xiong L, Kwon YM. Outcomes and Risk Factors Associated With Failures of Debridement, Antibiotics, and Implant Retention in Patients With Acute Hematogenous Periprosthetic Joint Infection. J Am Acad Orthop Surg. 2021;29(23):1024-30.

7. Courtney MW Jr, Snider TN, Cottrell DA. Dental implant placement in type II diabetics: a review of the literature. J Mass Dent Soc. 2010;59(1):12-4.

8. Kok TWK, Agrawal N, Sathappan SS, Chen WK. Risk Factors for Early Implant-Related Surgical Site Infection. Journal of Orthopaedic Surgery. 2016;24(1):72-6.

9. Pawar KB, Desai S, Bhonde RR, Bhole RP, Deshmukh AA. Wound with Diabetes: Present Scenario and Future. Curr Diabetes Rev. 2021;17(2):136-42.

10. Ahmed AS, Antonsen EL. Immune and vascular dysfunction in diabetic wound healing. J Wound Care. 2016;25(Sup7):35-46.

11. Moura J, Madureira P, Leal EC, Fonseca AC, Carvalho E. Immune aging in diabetes and its implications in wound healing. Clin Immunol. 2019;200:43-54.

12. Ochoa 0 , Torres FM, Shireman PK. Chemokines and diabetic wound healing. Vascular. 2007;15(6):350-5.

13. Pouget C, Dunyach-Remy C, Pantel A, Schuldiner S, Sotto A, Lavigne JP. Biofilms in Diabetic Foot Ulcers: Significance and Clinical Relevance. Microorganisms 2020, 8(10).

14. Shah SA, Sohail M, Khan S, Minhas MU, de Matas M, Sikstone V, Hussain Z, Abbasi M, Kousar M. Biopolymer-based biomaterials for accelerated diabetic wound healing: A critical review. Int J Biol Macromol. 2019;139:975-93.

15. Pinto AM, Cerqueira MA, Banobre-Lopes M, Pastrana LM, Sillankorva S. Bacteriophages for Chronic Wound Treatment: from Traditional to Novel Delivery Systems. Viruses 2020, 12(2).

16. Evelhoch SR. Biofilm and Chronic Nonhealing Wound Infections. Surg Clin North Am. 2020;100(4):727-32.

17. Feng X, Chen W, Ni X, Little PJ, Xu S, Tang L, Weng J. Metformin, Macrophage Dysfunction and Atherosclerosis. Front Immunol. 2021;12:682853. 
18. Buldak L, Labuzek K, Buldak RJ, Kozlowski M, Machnik G, Liber S, Suchy D, Dulawa-Buldak A, Okopien B. Metformin affects macrophages' phenotype and improves the activity of glutathione peroxidase, superoxide dismutase, catalase and decreases malondialdehyde concentration in a partially AMPK-independent manner in LPS-stimulated human monocytes/macrophages. Pharmacol Rep. 2014;66(3):418-29.

19. Chen M, Zhang J, Liu S, Zhou Z. [Effects of metformin on the polarization and Notch 1 expression of RAW264.7 macrophages]. Zhonghua Yi Xue Za Zhi. 2015;95(16):1258-61.

20. Ding C, Yang C, Cheng T, Wang X, Wang Q, He R, Sang S, Zhu K, Xu D, Wang J, et al. Macrophagebiomimetic porous Se@SiO2 nanocomposites for dual modal immunotherapy against inflammatory osteolysis. J Nanobiotechnology. 2021;19(1):382.

21. Samadzadeh S, Babazadeh M, Zarghami N, Pilehvar-Soltanahmadi Y, Mousazadeh H. An implantable smart hyperthermia nanofiber with switchable, controlled and sustained drug release: Possible application in prevention of cancer local recurrence. Mater Sci Eng C Mater Biol Appl. 2021;118:111384.

22. Pourpirali R, Mahmoudnezhad A, Oroojalian F, Zarghami N, Pilehvar Y. Prolonged proliferation and delayed senescence of the adipose-derived stem cells grown on the electrospun composite nanofiber co-encapsulated with TiO2 nanoparticles and metformin-loaded mesoporous silica nanoparticles. Int J Pharm. 2021;604:120733.

23. Zheng $\mathrm{H}$, Zuo B. Functional silk fibroin hydrogels: preparation, properties and applications. J Mater Chem B. 2021;9(5):1238-58.

24. Patil PP, Reagan MR, Bohara RA. Silk fibroin and silk-based biomaterial derivatives for ideal wound dressings. Int J Biol Macromol. 2020;164:4613-27.

25. Kim SH, Yeon YK, Lee JM, Chao JR, Lee YJ, Seo YB, Sultan MT, Lee OJ, Lee JS, Yoon SI, et al. Precisely printable and biocompatible silk fibroin bioink for digital light processing 3D printing. Nat Commun. 2018;9(1):1620.

26. Wu X, Zhou M, Jiang F, Yin S, Lin S, Yang G, Lu Y, Zhang W, Jiang X. Marginal sealing around integral bilayer scaffolds for repairing osteochondral defects based on photocurable silk hydrogels. Bioact Mater. 2021;6(11):3976-86.

27. Ribeiro M, Ferraz MP, Monteiro FJ, Fernandes MH, Beppu MM, Mantione D, Sardon H. Antibacterial silk fibroin/nanohydroxyapatite hydrogels with silver and gold nanoparticles for bone regeneration. Nanomedicine. 2017;13(1):231-9.

28. Shao J, Cui Y, Liang Y, Liu H, Ma B, Ge S. Unilateral Silver-Loaded Silk Fibroin Difunctional Membranes as Antibacterial Wound Dressings. ACS Omega. 2021;6(27):17555-65.

29. Youssef AM, Hasanin MS, El-Aziz MEA, Turky GM. Conducting chitosan/hydroxylethyl cellulose/polyaniline bionanocomposites hydrogel based on graphene oxide doped with Ag-NPs. Int J Biol Macromol. 2021;167:1435-44.

30. Ponomarev VA, Shvindina NV, Permyakova ES, Slukin PV, Ignatov SG, Sirota B, Voevodin AA, Shtansky DV. Structure and antibacterial properties of Ag-doped micropattern surfaces produced by 
photolithography method. Colloids Surf B Biointerfaces. 2019;173:719-24.

31. Stojkovska J, Zvicer J, Obradovic B. Preclinical functional characterization methods of nanocomposite hydrogels containing silver nanoparticles for biomedical applications. Appl Microbiol Biotechnol. 2020;104(11):4643-58.

32. Orecchioni M, Ghosheh Y, Pramod AB, Ley K. Macrophage Polarization: Different Gene Signatures in M1(LPS+) vs. Classically and M2(LPS-) vs. Alternatively Activated Macrophages. Front Immunol. 2019;10:1084.

33. Shapouri-Moghaddam A, Mohammadian S, Vazini H, Taghadosi M, Esmaeili SA, Mardani F, Seifi B, Mohammadi A, Afshari JT, Sahebkar A. Macrophage plasticity, polarization, and function in health and disease. J Cell Physiol. 2018;233(9):6425-40.

34. Zhou D, Huang C, Lin Z, Zhan S, Kong L, Fang C, Li J. Macrophage polarization and function with emphasis on the evolving roles of coordinated regulation of cellular signaling pathways. Cell Signal. 2014;26(2):192-7.

35. Ferrante CJ, Leibovich SJ. Regulation of Macrophage Polarization and Wound Healing. Adv Wound Care (New Rochelle). 2012;1(1):10-6.

36. Liu W, Yu M, Xie D, Wang L, Ye C, Zhu Q, Liu F, Yang L. Melatonin-stimulated MSC-derived exosomes improve diabetic wound healing through regulating macrophage $\mathrm{M} 1$ and $\mathrm{M} 2$ polarization by targeting the PTEN/AKT pathway. Stem Cell Res Ther. 2020;11(1):259.

37. Fadini GP, Menegazzo L, Rigato M, Scattolini V, Poncina N, Bruttocao A, Ciciliot S, Mammano F, Ciubotaru CD, Brocco E, et al. NETosis Delays Diabetic Wound Healing in Mice and Humans. Diabetes. 2016;65(4):1061-71.

38. Wong SL, Demers M, Martinod K, Gallant M, Wang Y, Goldfine AB, Kahn CR, Wagner DD. Diabetes primes neutrophils to undergo NETosis, which impairs wound healing. Nat Med. 2015;21(7):815-9.

39. Speziale P, Pietrocola G. Staphylococcus aureus induces neutrophil extracellular traps (NETs) and neutralizes their bactericidal potential. Computational Structural Biotechnology Journal. 2021;19:3451-7.

40. Hoste E, Maueroder C, van Hove L, Catrysse L, Vikkula HK, Sze M, Maes B, Karjosukarso D, Martens L, Goncalves A, et al: Epithelial HMGB1 Delays Skin Wound Healing and Drives Tumor Initiation by Priming Neutrophils for NET Formation. Cell Rep 2019, 29(9):2689-2701 e2684.

41. Huang W, Jiao J, Liu J, Huang M, Hu Y, Ran W, Yan L, Xiong Y, Li M, Quan Z, et al. MFG-E8 accelerates wound healing in diabetes by regulating "NLRP3 inflammasome-neutrophil extracellular traps" axis. Cell Death Discov. 2020;6:84.

42. Jorch SK, Kubes P. An emerging role for neutrophil extracellular traps in noninfectious disease. Nat Med. 2017;23(3):279-87.

43. Conforti A, Wahlers T, Paunel-Gorgulu A. Neutrophil extracellular traps modulate inflammatory markers and uptake of oxidized LDL by human and murine macrophages. PLoS One. 2021;16(11):e0259894. 
44. Griffiths HR, Gao D, Pararasa C. Redox regulation in metabolic programming and inflammation. Redox Biology. 2017;12:50-7.

45. Abdalla M, Goc A, Segar L, Somanath PR. Akt1 mediates alpha-smooth muscle actin expression and myofibroblast differentiation via myocardin and serum response factor. $\mathrm{J}$ Biol Chem. 2013;288(46):33483-93.

46. Kim TH, Cho HY, Lee SM. High-voltage pulsed current stimulation enhances wound healing in diabetic rats by restoring the expression of collagen, alpha-smooth muscle actin, and TGF-beta1. Tohoku J Exp Med. 2014;234(1):1-6.

\section{Scheme}

Scheme 1 is available in supplementary section.

\section{Figures}

\section{Figure 1}

Characterization of the synthesized nanoparticles. SEM micrographs of (a) MSN and (b) MET@MSNs

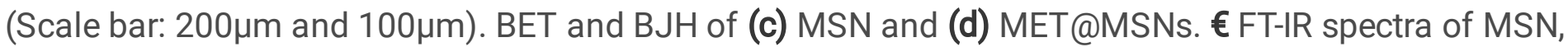
Met, and MET@MSNs. 
a.

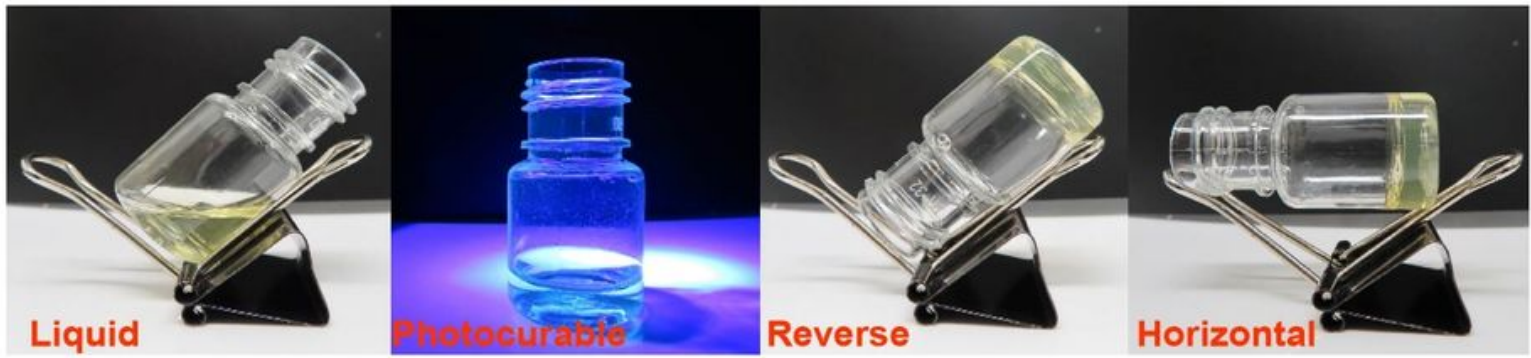

b.

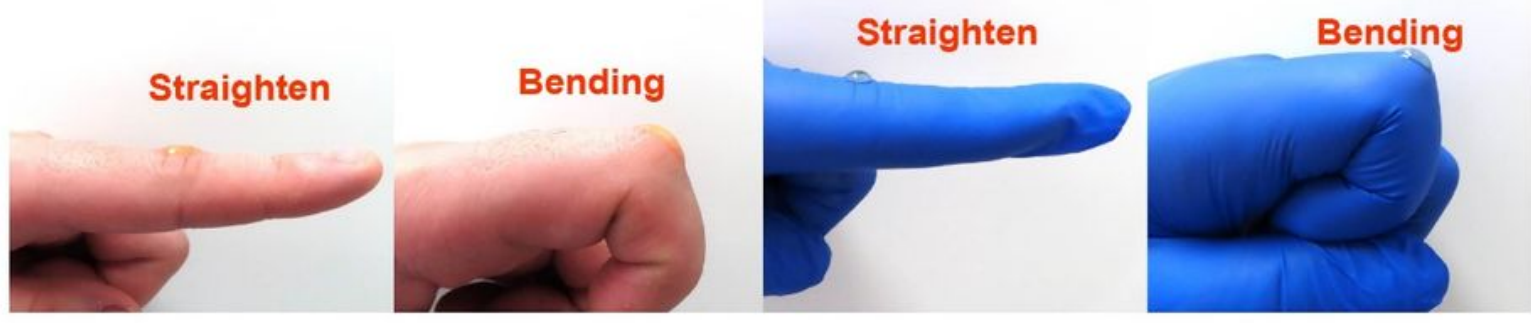

c.
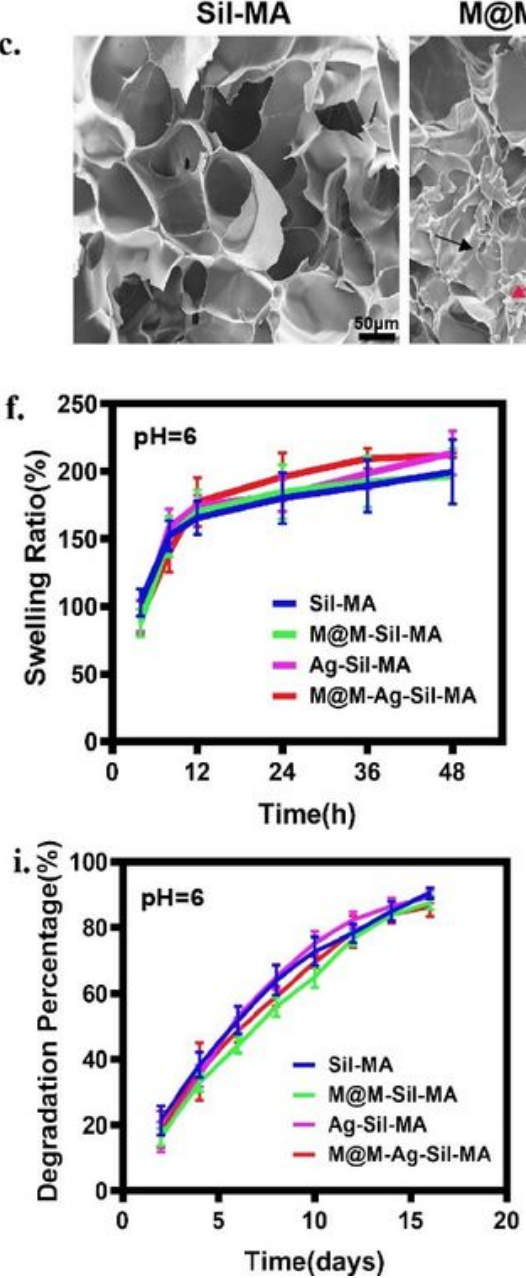

M@M-Ag-Sil-MA

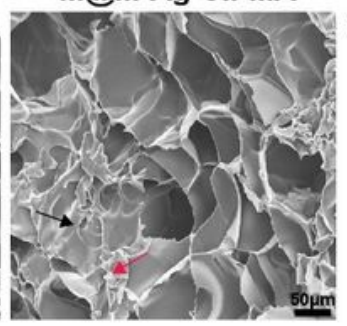

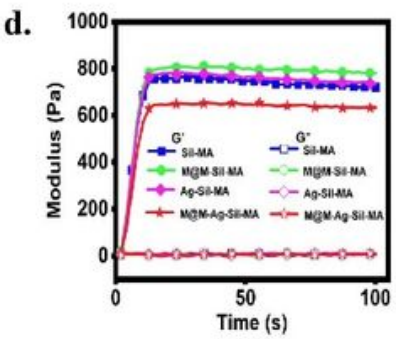
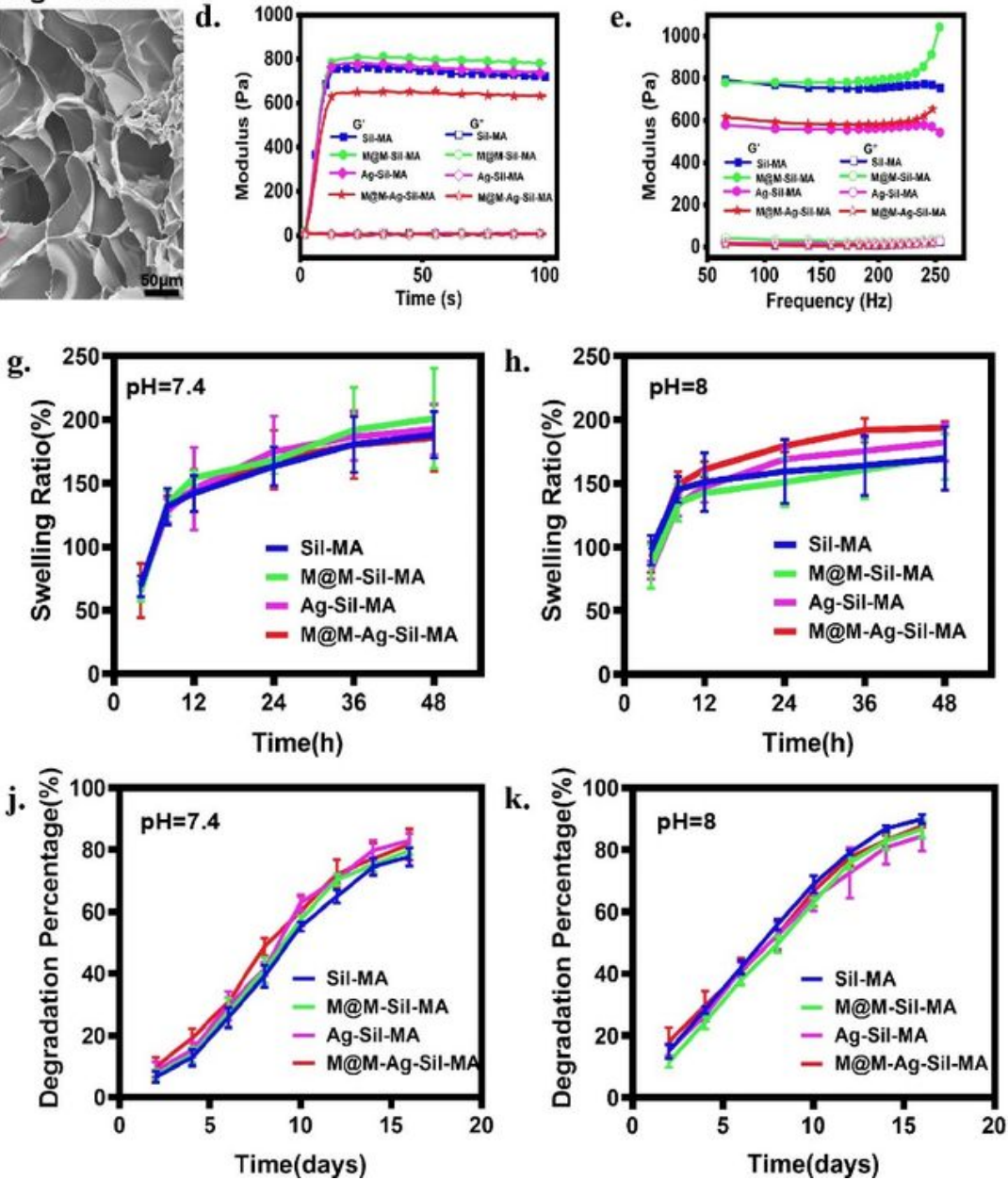

Figure 2

Characterization of the hydrogel system. (a) Photographs of the solution to hydrogel transition by photocuring. (b) Adhesion and bending ability of the hydrogel system on different surfaces. (b Representative SEM images of the hydrogel (Scale bar: $50 \mu \mathrm{m})$. The black and pink arrows represent MET@MSNs and Ag NPs encapsulated in hydrogels, respectively. Storage modulus at different (d) time $\mathrm{a} €(\mathbf{e})$ frequencies. (f-h) Swelling property of the hydrogel system under different $\mathrm{pH}$ values $(\mathrm{pH}=$ 
6.0/7.4/8.0). (i-k) Degradation property of the hydrogel system under different $\mathrm{pH}$ values $(\mathrm{pH}=$ $6.0 / 7.4 / 8.0)$.
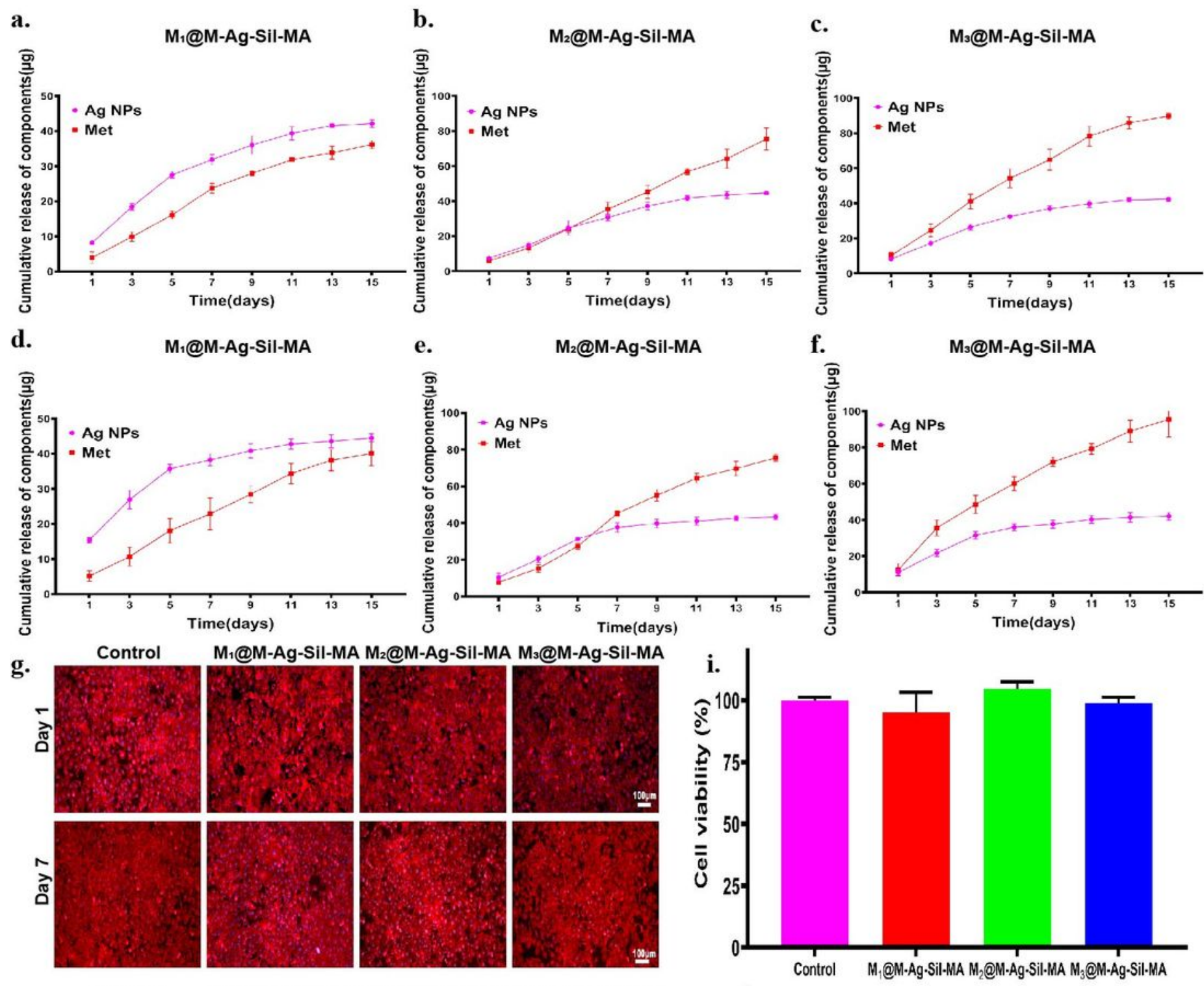

h.
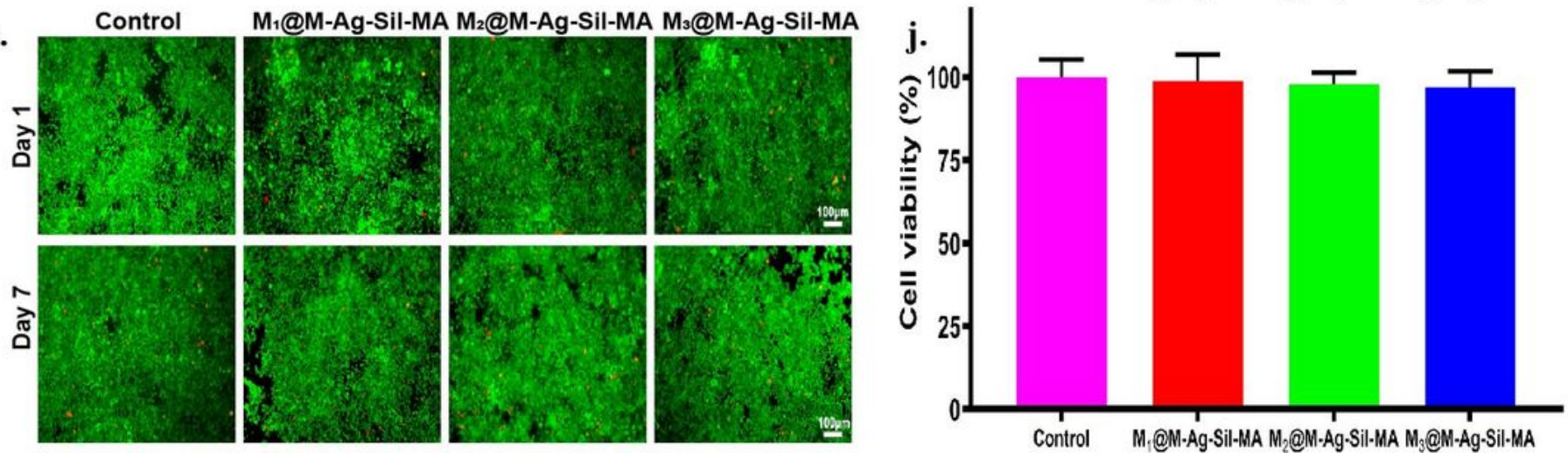

Figure 3

Drug release and biocompatibility assay of the hydrogel system in vitro. Cumulative release curves of Met and Ag NPs of hydrogel systems with different Met and Ag NPs mass ratios (1:1, 2:1, and 3:1) in neural 
$(\mathrm{pH}=7.4)(\mathrm{a}-\mathrm{c})$ and acidic $(\mathrm{pH}=6.0)(\mathrm{d}-\mathrm{f})$ medium. ( $\mathrm{g})$ Representative fluorescence microscopy of Ea.hy

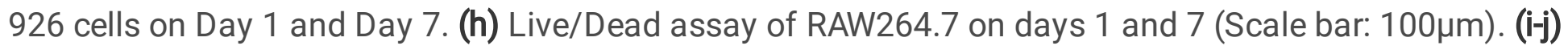
Biocompatibility of L929 cells by CCK8 assay on days 1 and 7 .

a.

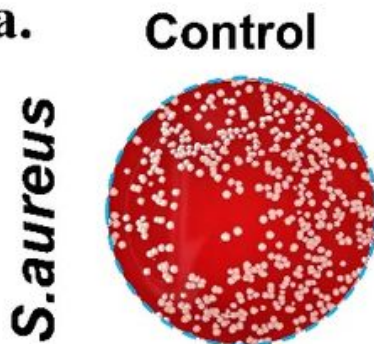

Sil-MA

M@M-Sil-MA
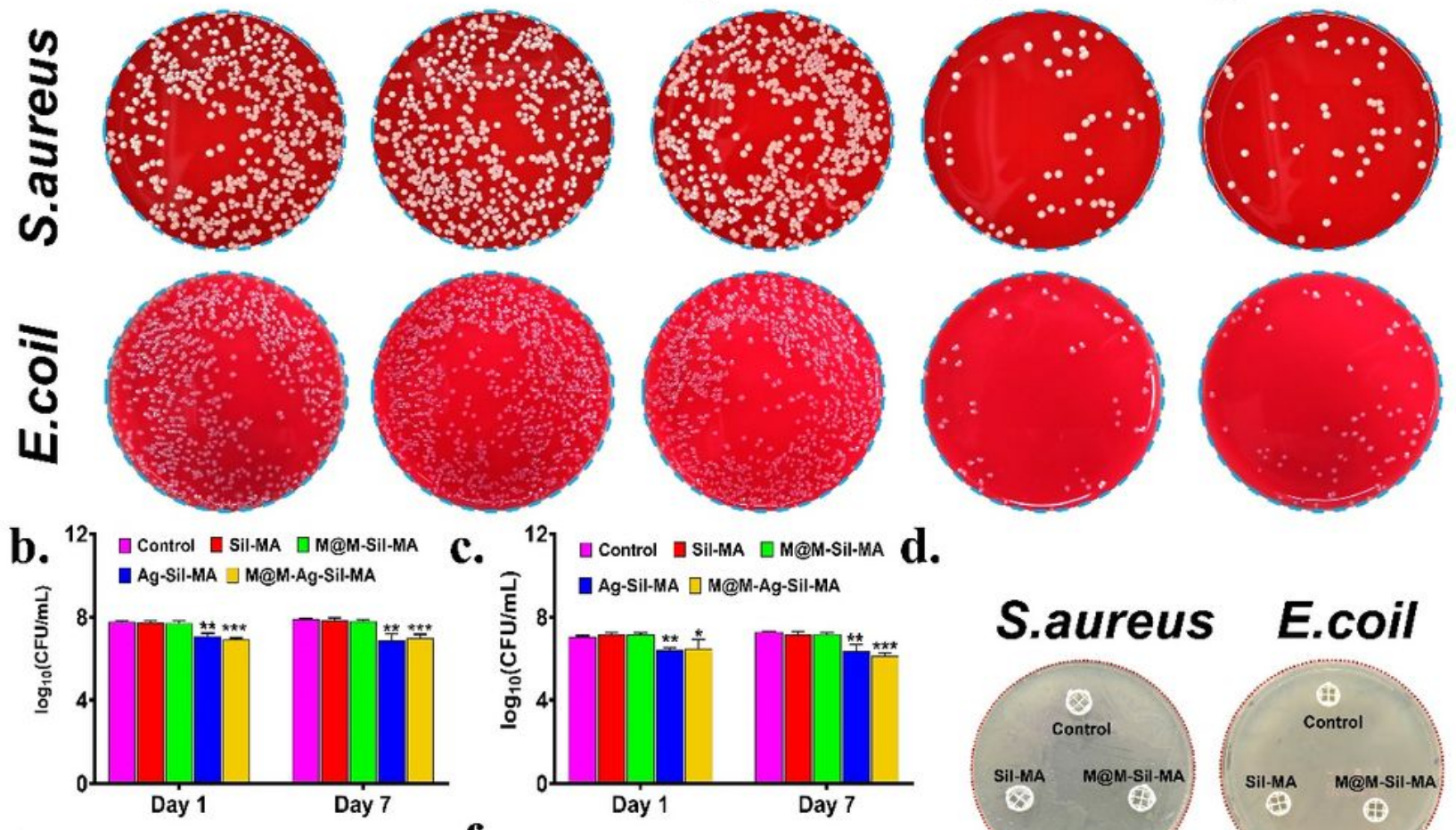

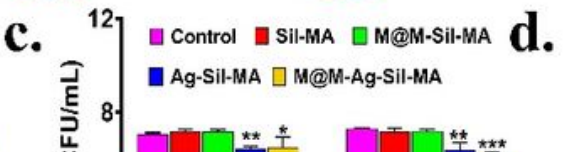

S.aureus E.coil

e.

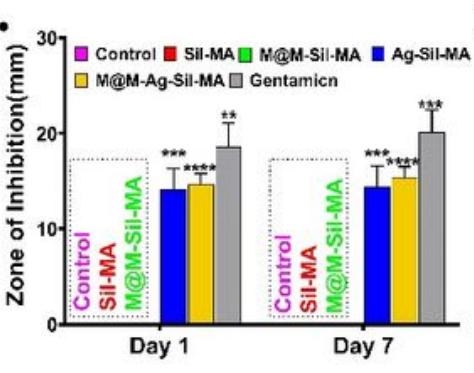

f.
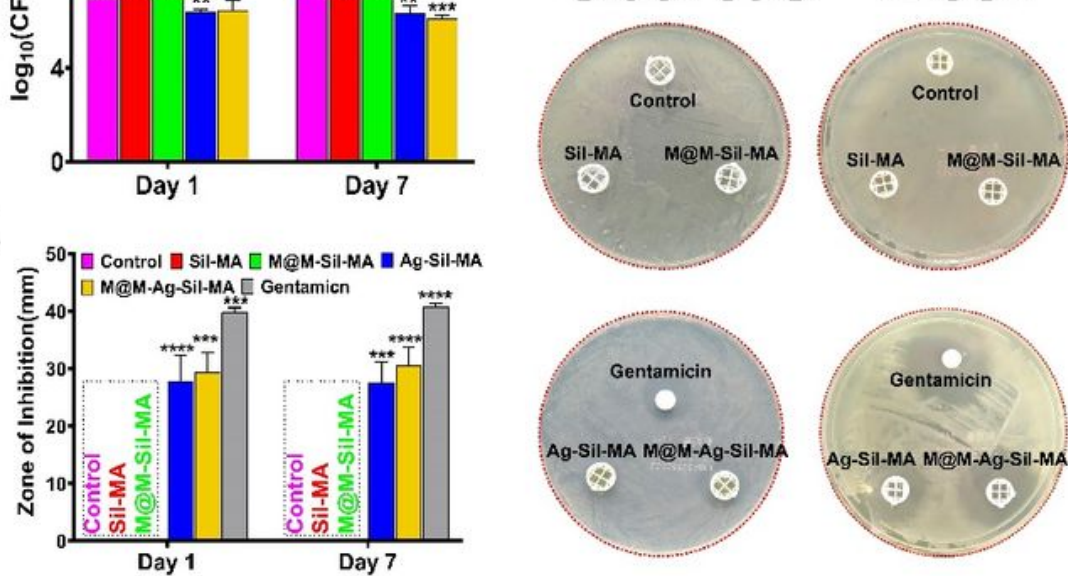

g.

Control

Sil-MA
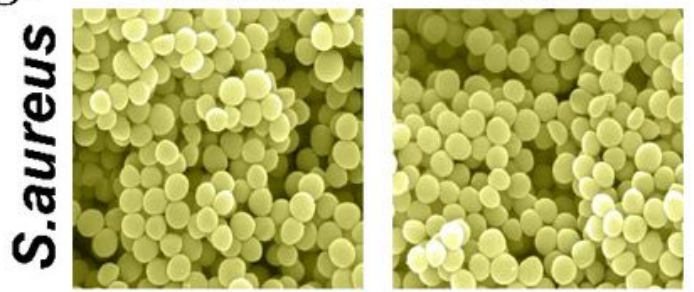

M@M-Sil-MA

Ag-Sil-MA M@M-Ag-Sil-MA
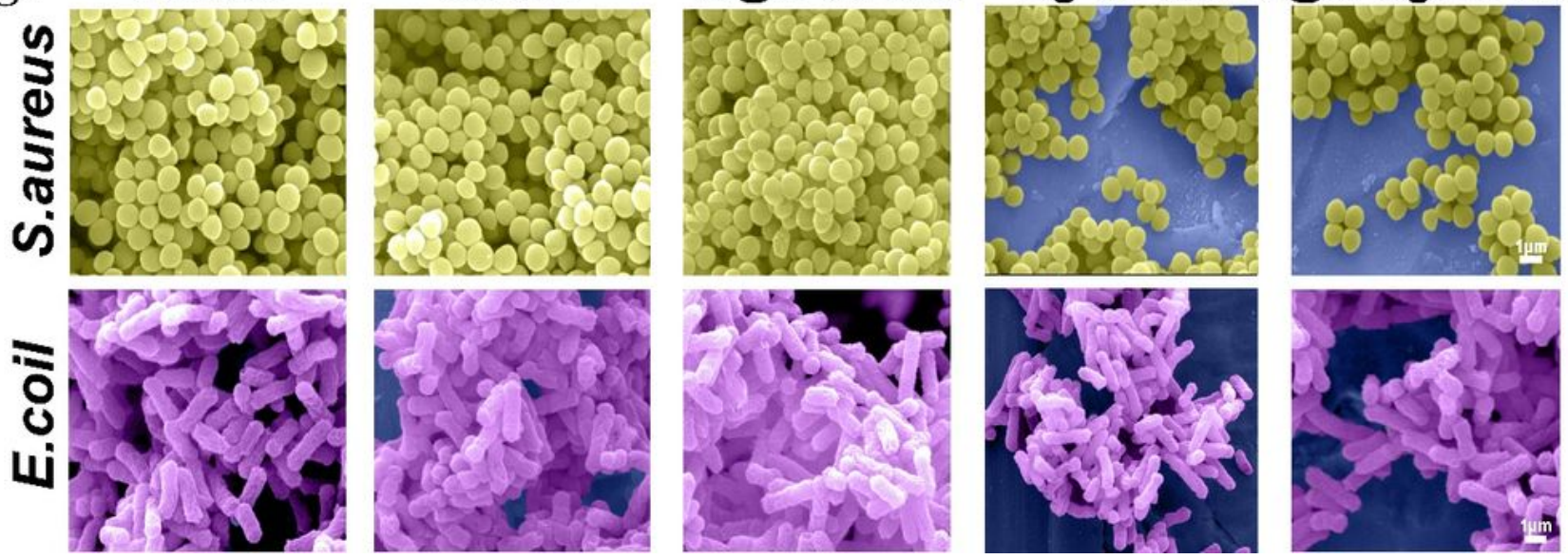

Figure 4 
Antibacterial properties of the hydrogel systems. (a) Representative culture images of bacterial colonies and the relative amounts of the corresponding colonies of $S$. aureus (b) and E. coli (c). (d) Representative photos of the hydrogel systems and Gentamicin disks of ZOls against $S$. aureus and E. coli and the relative diameters of corresponding ZOls of $S$. aureus (e) and E. coli (f). (g) Representative SEM images of bacteria. Yellow spheres indicate $S$. aureus, and violet rods indicate $E$. coli. (Note: ${ }^{*} \mathrm{P}<0.05, * \star \mathrm{P}<0.01$ and $\left.{ }^{\star * * P}<0.001\right)($ Scale bar: $1 \mu \mathrm{m})$
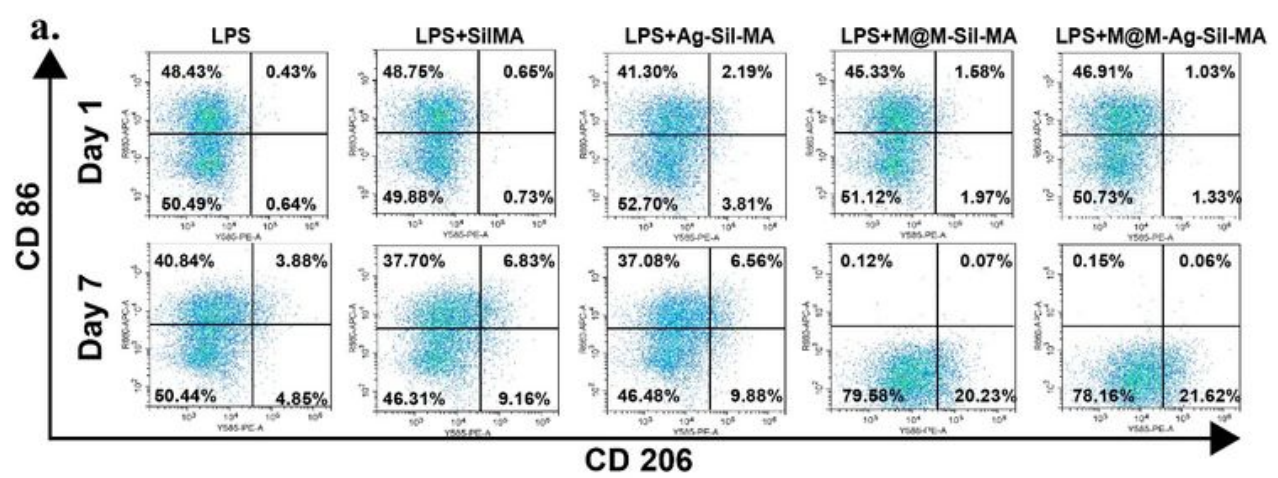

b.
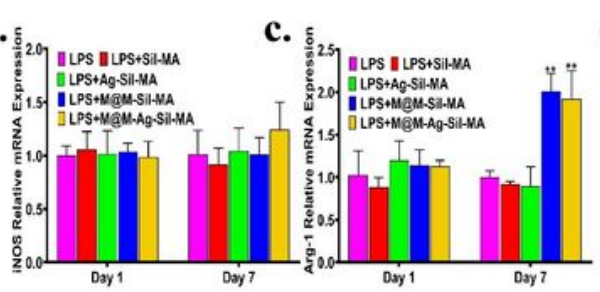

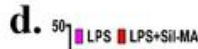

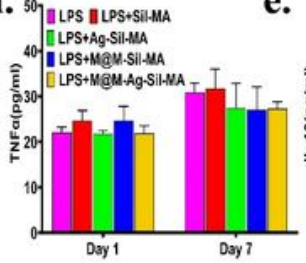

e. ${ }^{150}$ [IPS ILPS+51HAA

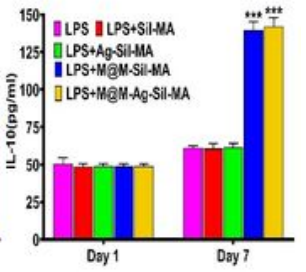

f.

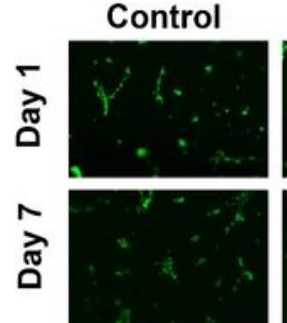

Sil-MA
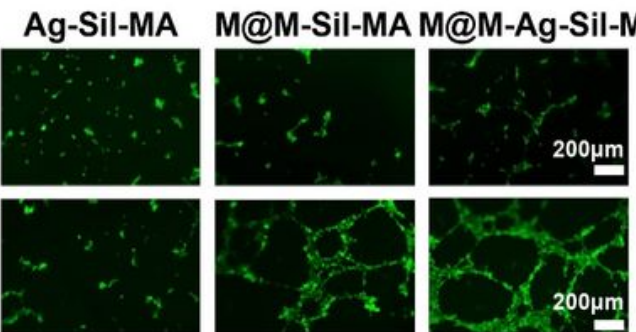

g.
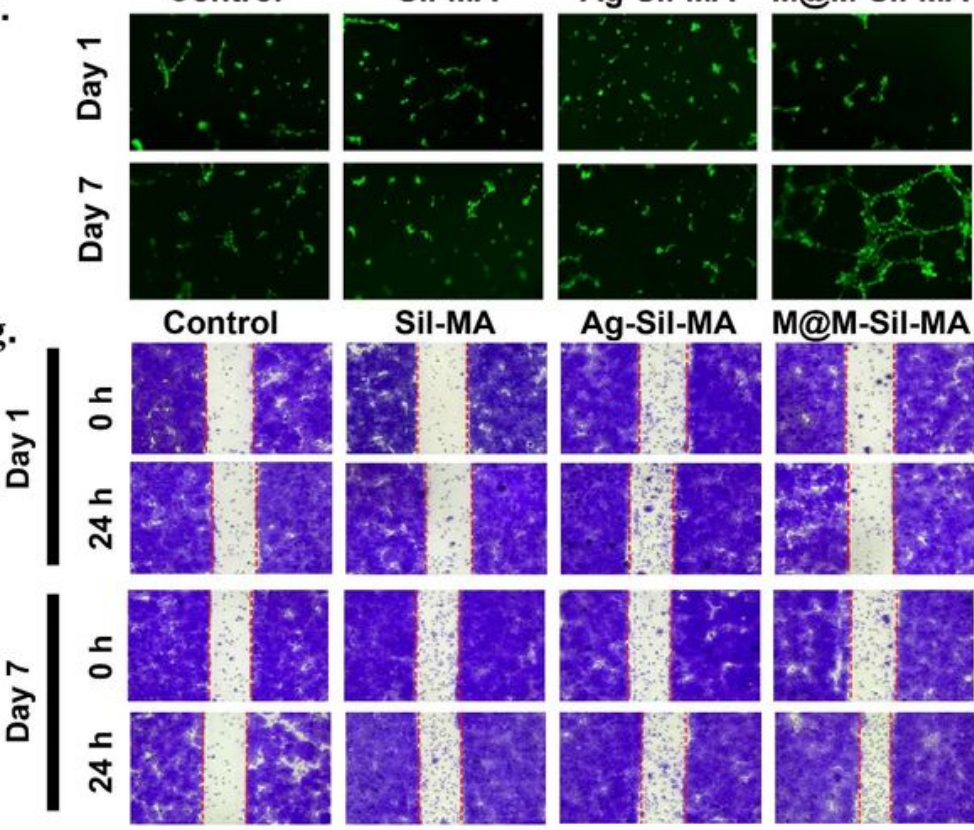

Ag-Sil-MA M@M-Sil-MA M@M-Ag-Sil-MA
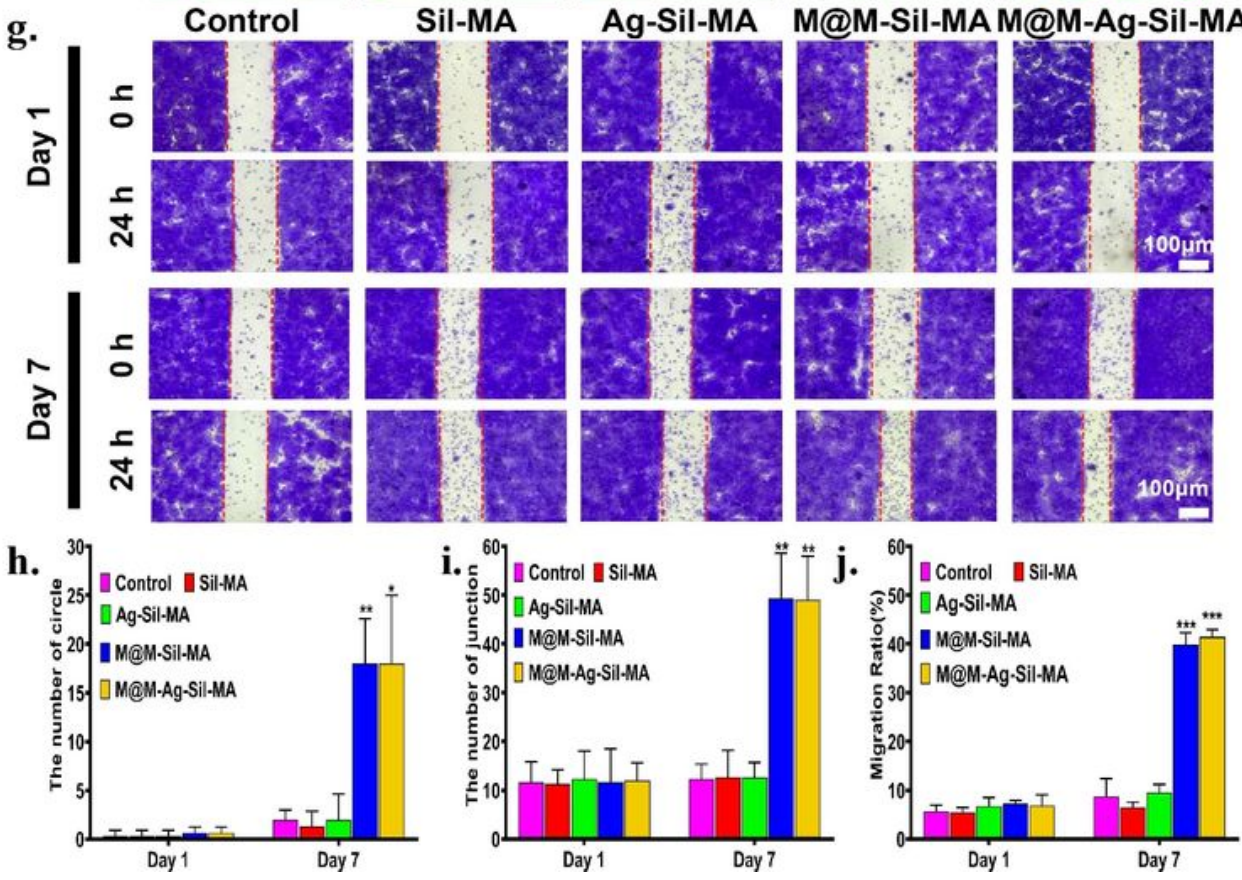


\section{Figure 5}

Immunomodulatory function of macrophages in vitro. (a) Representative flow cytometry results after 1 and $7 \mathrm{~d}$ of co-culture with CMs of hydrogel systems of RAW264.7. Percentages of CD86- and CD206positive cells. (b, c) Expression of M1-related gene, iNOS, and M2-related genes, Arg-1 on days 1 and 7. (d, e) ELISA results for TNF- $a$ and IL-10. (f) Tube formation pictures of Ea.hy 926 cells on days 1 and 7. (Scale bar: $200 \mu \mathrm{m})$. (g) Pictures of migration of L929 cells on days 1 and 7. (Scale bar: $100 \mu \mathrm{m})$. (h, i) Quantitative results of tube formation. (j) Quantitative results of cell migration. (Note: ${ }^{*} P<0.05,{ }^{* *} \mathrm{P}<$ 0.01 and $* * * P<0.001)$ 
a.
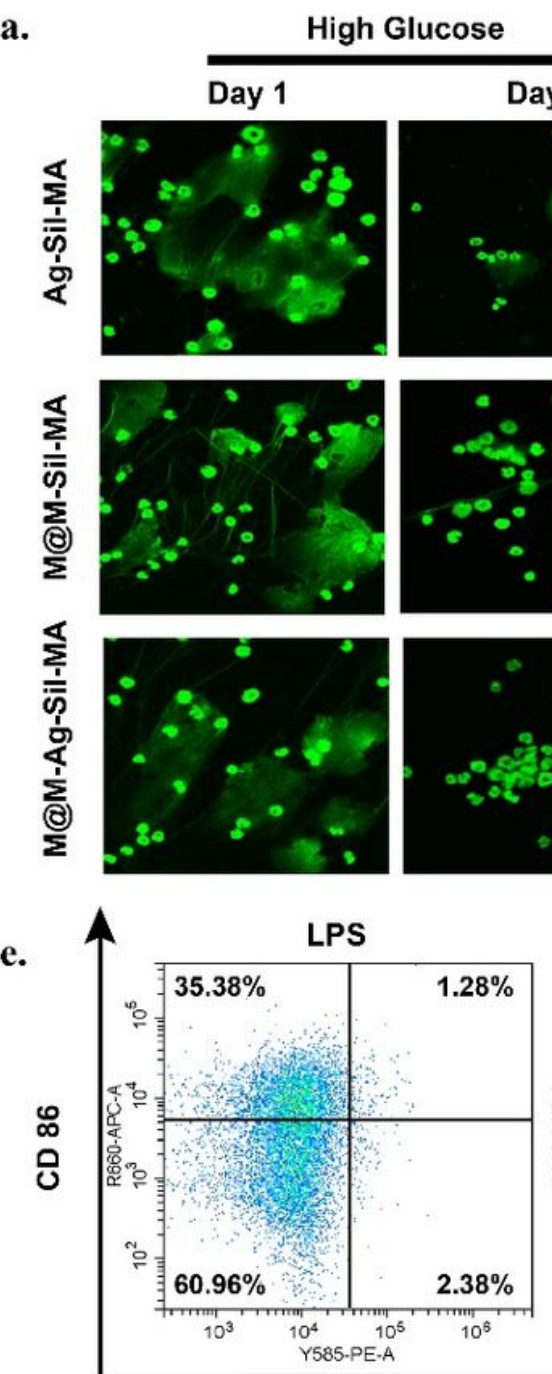

Day 7
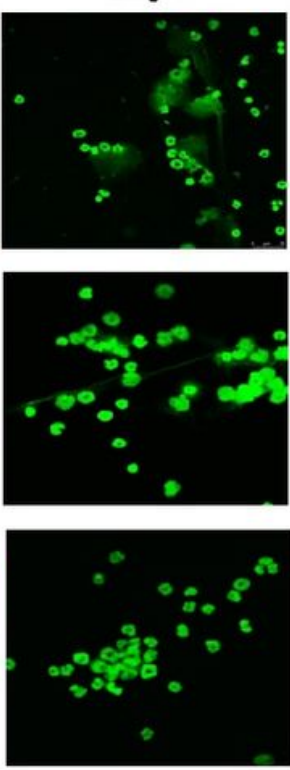

LPS

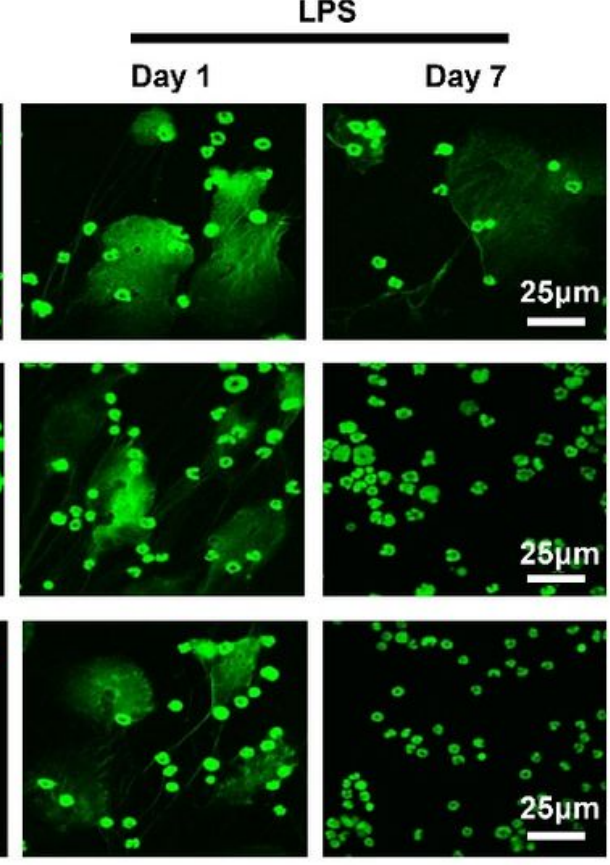

b.

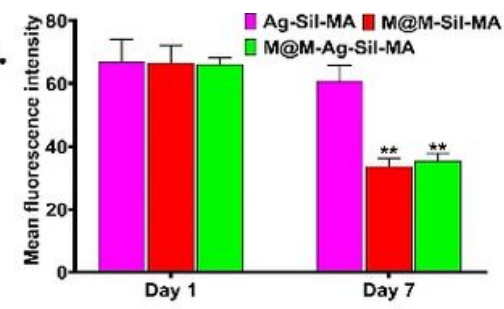

c. ${ }^{5000}$ ] Ag-Sil-MA $\square \mathrm{M@M-Sil-MA}$

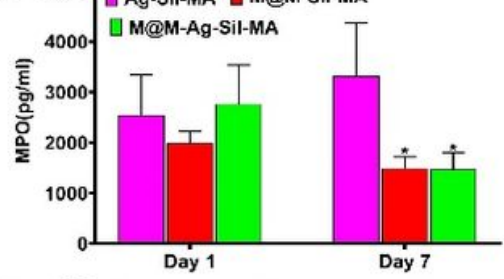

d. $\left.{ }^{300}\right] \|$ Ag-Sil-MA $\| \mathrm{M} @ M-S i l-M A$

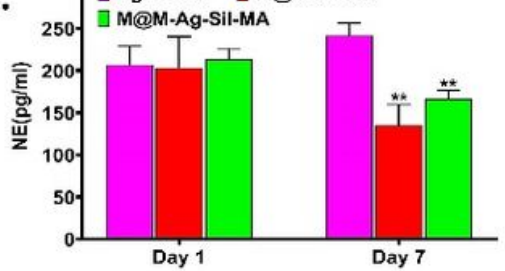

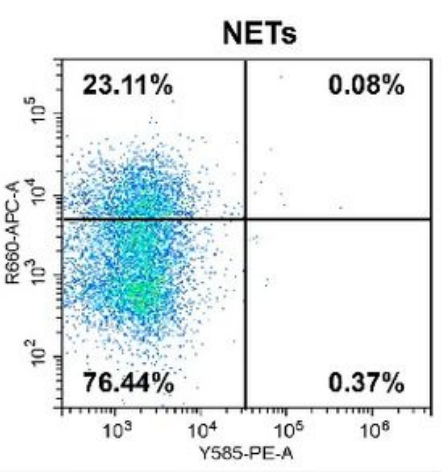
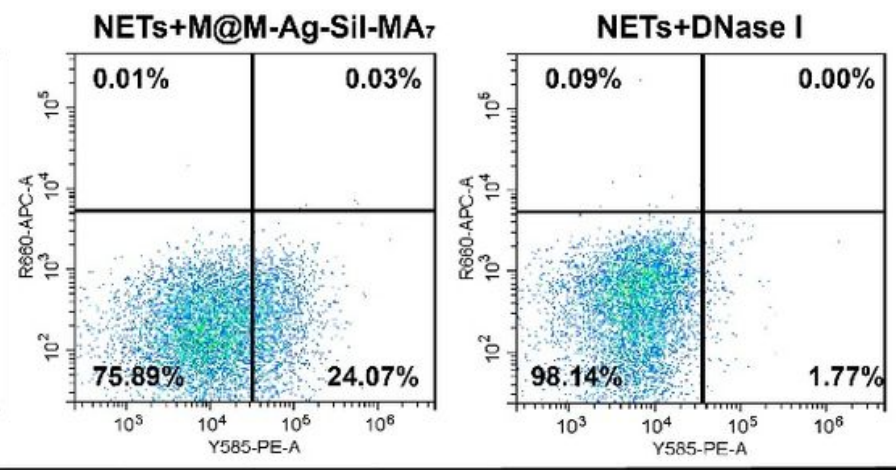

\section{206}
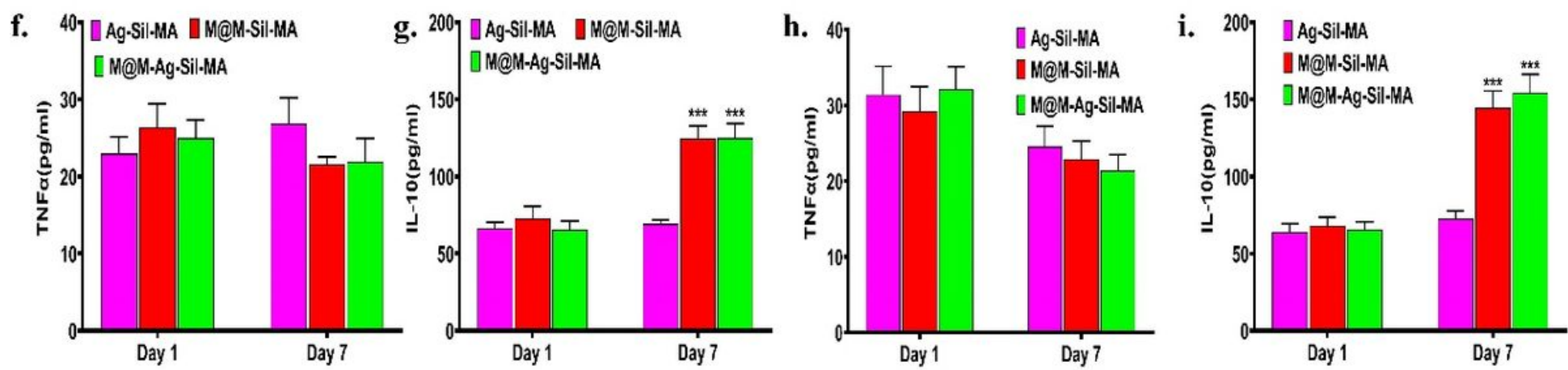

Figure 6

Immunomodulation of NETs in vitro. (a) Fluorescence micrographs of high glucose (50 mM)-treated and LPS-treated neutrophils stained with SYTOX green on Day 1 and Day 7 (scale bar: $25 \mu \mathrm{m}$ ).

(b)Fluorescence intensity of NET high glucose-treated neutrophils. (c, d) ELISA results for MPO and NE of NETs of high glucose-treated neutrophils. (e) Representative flow cytometry results after co-culture with LPS, extracts of NETs, extracts of hydrogel systems, or DNase I of RAW264.7. Percentages of CD86- and 
CD206-positive cells. (f-i) ELISA results for TNF- $a$ and IL-10 of high glucose-treated and LPS-treated neutrophils.

a.

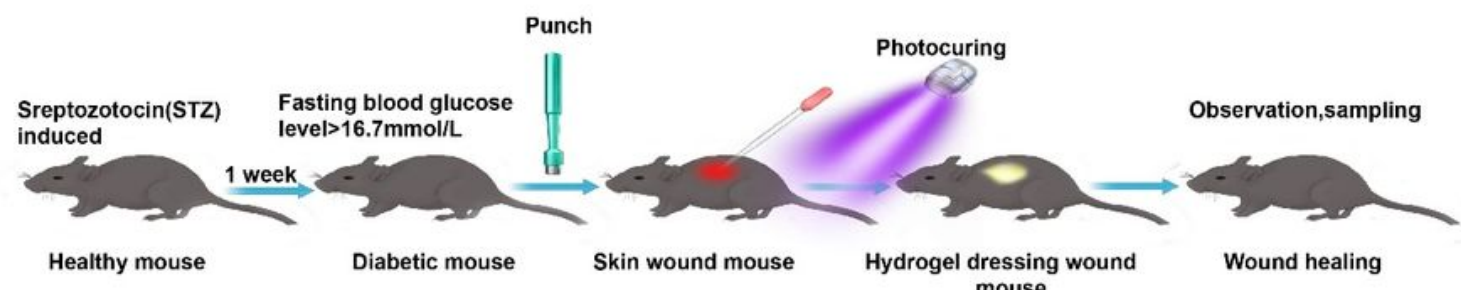

b.
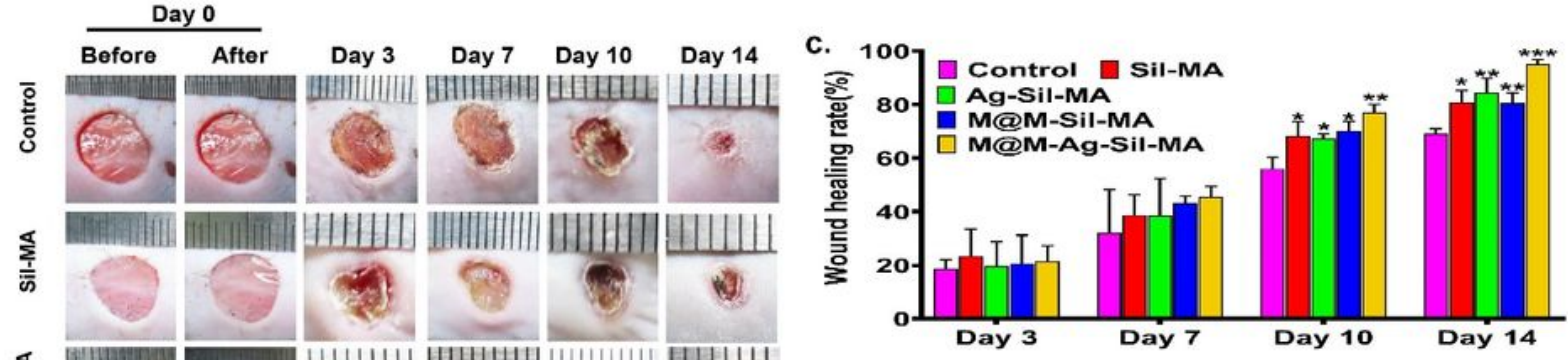

e.
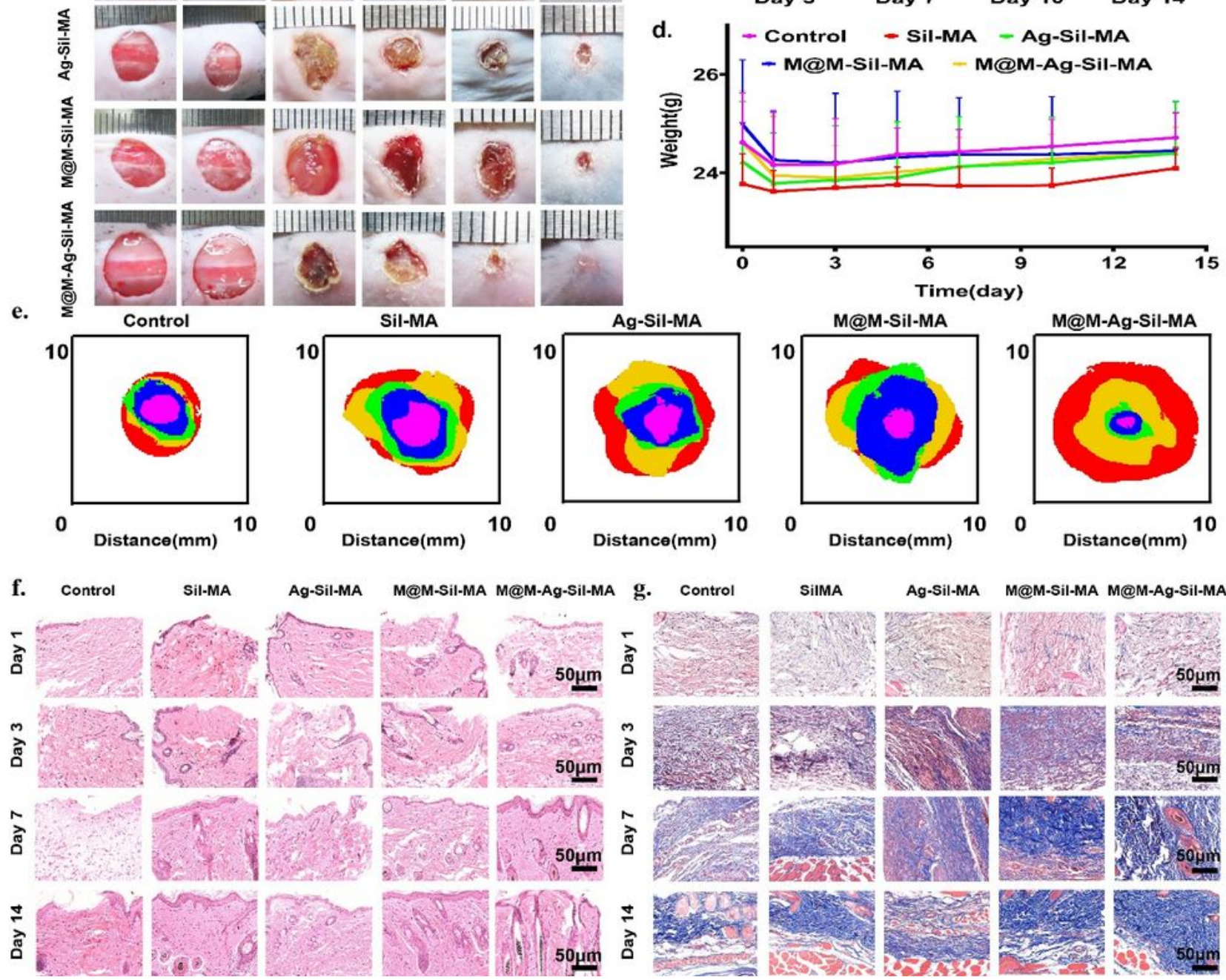

Figure 7

Diabetic wound healing in vivo. (a) Schematic diagram of diabetic wound and in situ hydrogel system photocuring. (b) Photographs of the wounds with different treatments on days 0 (before and after 
photocuring), 3, 7, 10 and 14. (c) Skin wound healing rate of the diabetic wound model at different time points. (d) Weight of the diabetic mice at different time points. (e) The overlaid images of the wound boundary at different time points. (f) H\&E staining on days 1, 3, 7, and 14. (g) Masson's trichrome staining on days $1,3,7$, and 14 . (scale bar: $50 \mu \mathrm{m}$ )

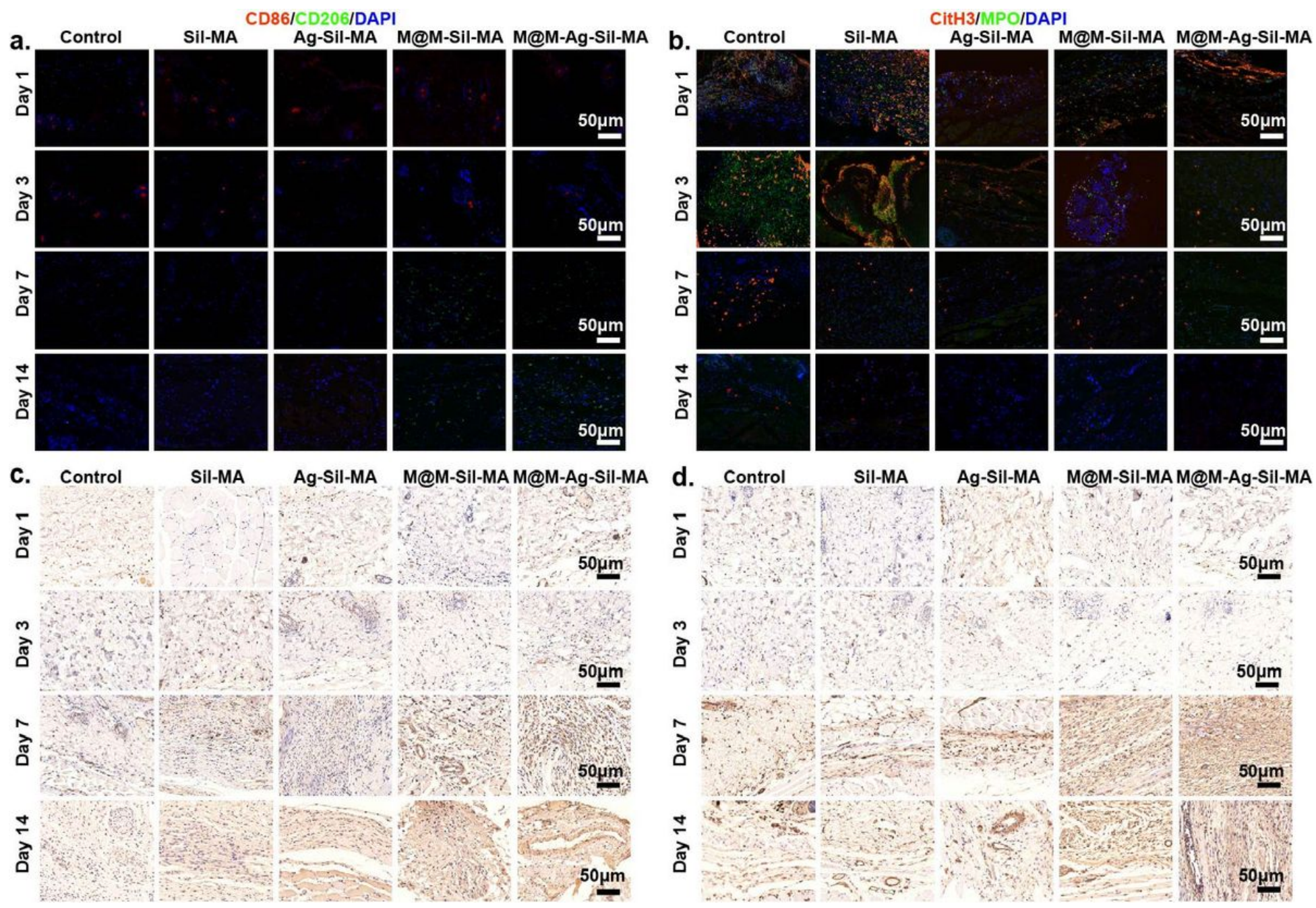

Figure 8

Immunomodulation of macrophages and NETs and tissue regeneration in vivo. (a) Immunofluorescence

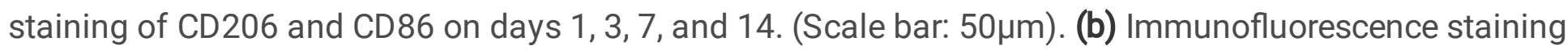

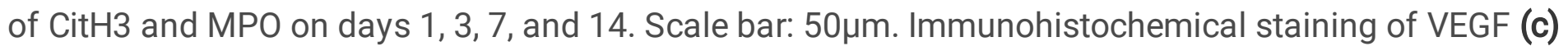

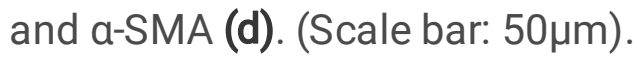

\section{Supplementary Files}

This is a list of supplementary files associated with this preprint. Click to download.

- floatimage1.png

- SI.pptx 